\title{
Electrocorticography is superior to subthalamic local field potentials for movement decoding in Parkinson's disease
}

Timon Merk ${ }^{1}$, Victoria Peterson ${ }^{2,5}$, Witold Lipski ${ }^{3}$, Benjamin Blankertz ${ }^{4}$, Robert S. Turner ${ }^{3}$, Ningfei Li ${ }^{1}$, Andreas Horn ${ }^{1}$, Andrea A. Kühn ${ }^{1}$, R. Mark Richardson ${ }^{2,5 *}$, Wolf-Julian Neumann ${ }^{1 *}$

${ }^{1}$ Movement Disorder and Neuromodulation Unit, Department of Neurology, Charité - Universitätsmedizin Berlin, Berlin, Berlin, 10117, Germany

${ }^{2}$ Brain Modulation Lab, Department of Neurosurgery, Massachusetts General Hospital, Boston, Massachusetts, 02114, USA

${ }^{3}$ Department of Neurobiology, University of Pittsburgh, Pittsburgh, Pennsylvania, 15213, USA

${ }^{4}$ Department of Computer Science, Technische Universität Berlin, Berlin, Berlin, 10587, Germany

${ }^{5}$ Harvard Medical School, Boston, Massachusetts, 02114, USA

*These authors contributed equally to this work

Corresponding author and Lead contact: Wolf-Julian Neumann, MD, Assistant Professor for Interventional and Cognitive Neuromodulation, Movement Disorder and Neuromodulation Unit, Charité Universitätsmedizin Berlin, Chariteplatz 1, 10117 Berlin, julian.neumann@charite.de

Timon Merk: timon.merk@charite.de

Victoria Peterson: vpeterson2@mgh.harvard.edu

Witold Lipski: lipskiw@upmc.edu

Benjamin Blankertz: benjamin.blankertz@tu-berlin.de

Robert Sterling Turner: rturner@pitt.edu

Ningfei Li: Ningfei.li@charite.de

Andreas Horn: andreas.horn@charite.de

Andrea Kühn: andrea.kuehn@charite.de

Robert Mark Richardson: Mark.Richardson@mgh.harvard.edu

Wolf-Julian Neumann: julian.neumann@charite.de 


\section{Summary}

Smart brain implants will revolutionize neurotechnology for improving the quality of life in patients with brain disorders. The treatment of Parkinson's disease (PD) with neural implants for deep brain stimulation (DBS) presents an avenue for developing machine-learning based individualized treatments to refine human motor control. We developed an optimized movement decoding approach to predict grip-force based on sensorimotor electrocorticography (ECoG) and subthalamic local field potentials in PD patients undergoing DBS neurosurgery. ECoG combined with Bayesian optimized extreme gradient boosted decision trees outperformed multiple state of the art machine learning approaches. We further developed a whole brain connectomics approach to predict decoding performance in invasive neurophysiology, relevant for connectomic targeting of distributed brain networks for neural decoding. PD motor impairment deteriorated decoding performance, suggestive of a role for dopamine in human movement coding capacity. Our study provides an advanced neurophysiological and computational framework to aid development of intelligent adaptive DBS.

\section{Keywords}

Parkinson's disease, Deep brain stimulation, Machine learning, Neuromodulation, XGBOOST, Basal ganglia, Electrocorticography, Local field potentials, Oscillations 


\section{Introduction}

Subthalamic deep brain stimulation (DBS) for Parkinson's disease (PD) is one of the most successful neurotechnological advances in translational neuroscience to date. In addition to its clinical utility, DBS has provided unique insight into the neurophysiology of movement disorders (Cagnan et al., 2019; Krauss et al., 2021; Kühn and Volkmann, 2017). Here, the parkinsonian hypodopaminergic state has been associated with increased beta synchronization in the basal ganglia (Kehnemouyi et al., 2021; Kühn et al., 2006; Neumann et al., 2016b) and exaggerated phase amplitude coupling and waveform sharpness asymmetry in cortex (Cole et al., 2017; De Hemptinne et al., 2015). Symptom severity in the OFF medication state was shown to correlate with beta power in the STN across patients (Kühn et al., 2006; Neumann et al., 2016a). Such observations have inspired the idea of adaptive closed-loop DBS (aDBS), where electrophysiological signals are used to change stimulation parameters in response to evolving clinical states (Beudel and Brown, 2016; Hwang et al., 2020; Little et al., 2013; Petrucci et al., 2020; Piña-Fuentes et al., 2019; Swann et al., 2018; Tinkhauser et al., 2017; Velisar et al., 2019). In a series of seminal papers it was shown that significant clinical benefit and reduced side-effects could be achieved, when stimulation was triggered by beta power (Arlotti et al., 2016; Little et al., 2013; Velisar et al., 2019). In the future, neurotechnological treatments may have the potential to outperform traditional drug regimes, due to a key advantage in the temporal (and spatial) precision of therapeutic delivery. Treatment can be aligned to changes of therapeutic demand, paving the way for a precision medicine approach toward individualized symptom-specific DBS (He et al., 2020; Neumann and Rodriguez-Oroz, 2021; Neumann et al., 2019a; Opri et al., 2020a). To make sensing algorithms more deliberate and specific (instead of mere beta power estimation), machine-learning can integrate multivariate feature sets for adaptive DBS control. In movement disorders, decoding of movement kinematics could inform stimulation about the patients' ability and intention to move, exactly at the time when therapeutic intervention is needed (Neumann et al., 2019a). To this date, movement has been primarily decoded from cortical signals (Leuthardt et al., 2004; Opri et al., 2020b; Schalk et al., 2007; Xie et al., 2018) but recent publications have shown promising results using local field potentials (LFP) from subthalamic DBS electrodes (Khawaldeh et al., 2020; Shah et al., 2018; Tan et al., 2019).

In the present study, we aimed at decoding continuous grip-force by combining state-of-art machine learning algorithms with multimodal invasive neurophysiology and whole-brain connectomics in patients with Parkinson's disease. Our results address the utility of cortical vs. subcortical signals to accurately decode grip-force and establish a link between motor system encoding capacity and dopaminergic depletion in PD. Finally, decoding performance of cortical recording locations could be predicted based on structural and functional connectivity fingerprints seeding from recording locations. Together, this 
multimodal connectomic decoding framework will impact $\mathrm{BCl}$ implant strategies beyond movement disorders.

\section{Results}

\section{Real-time processing \& Feature Definition}

We analyzed sensorimotor electrocorticography and subthalamic local field potential data recorded intraoperatively during performance of a grip force task from 11 patients with Parkinson's disease, undergoing DBS implantation (Figure 1A). Individual electrode localizations in montreal neurological institute (MNI) space are shown in Figure 1B. Figure 1C shows the well-characterized pattern of increased gamma band and decreased alpha and beta band activity resulting from movement related spectral changes in both sensorimotor ECoG and subthalamic LFP (Androulidakis et al., 2007; Kondylis et al., 2016; Kühn et al., 2004; Lofredi et al., 2018) aligned to onset of grip force (total $n=2685$, on average $n=244 \pm 149$ STD trials per patient). For the use in machine learning models band power feature time-series were extracted in a real-time $\mathrm{BCl}$ compatible implementation, for which at each sample only information from previous samples are available (Figure 1D; i.e. no smoothing, windowing, spectral decomposition or normalization that extended information content beyond each sample were used in the input feature vectors). Therefore, the signal was streamed in virtual packets of 100 ms length at a sampling rate of $2000 \mathrm{~Hz}$ (400 samples) to mimic the real-time online application. Variance as a measure of amplitude of rereferenced, band-pass filtered raw data segments was extracted at $10 \mathrm{~Hz}(10$ band-power features per second) with an adaptive window length from $1000-100 \mathrm{~ms}$ of past data for eight oscillatory features $[\theta(4-8 \mathrm{~Hz}), \alpha(8-12 \mathrm{~Hz}), \beta(13-35 \mathrm{~Hz})$, low $\beta(13-20 \mathrm{~Hz})$, high $\beta(20-35 \mathrm{~Hz})$, all $\gamma$ $(60-200 \mathrm{~Hz}), \mathrm{Y}(60-80 \mathrm{~Hz})$ and high frequency activity (HFA) $(90-200 \mathrm{~Hz})]$. All features were normalized to the median of the past 10 seconds to ensure sustainable performance in the presence of slow shifts in spectral activation patterns e.g. due to changes in wakefulness, impedance or medication. Note, that no visible changes of this kind were present in the acute dataset. The target variable was the continuously measured grip-force (z-scored for each recording session), which was cleaned from noise and baseline drift (Xie et al., 2018). Thus, every sample of input features was paired with a target sample of ground-truth grip-force measurement, regardless whether movement was present or absent. A high performance was achieved when the trained models predicted no or low grip-force in the absence of movement and correct estimates of grip-force during movement, as evaluated with the $R^{2}$ coefficient of determination. 


\section{Performance saturation occurs at a time concatenation of $500 \mathrm{~ms}$}

A linear model analysis all eight oscillatory features per channel was used to investigate the resulting contributing band correlations for time-points preceding target samples of continuous grip-force measurements within a one second window. Note that ECoG strip designs varied slightly between patients, leading to varying dimensions of overall input feature matrices. Furthermore, the number of ECoG channels (average $n=+-$ STD per hemisphere) is higher compared to the number of STN LFP channels $(n=3)$. Figure $2 A$ shows average weight distributions for the best performing channels per electrode. Negative weights were learned for $\beta$, and positive weights for $\gamma$ bands. A linear model was trained using each single time-point and one respective band and the weight distribution of the best performing channels for ECoG and STN for contra- and ipsilateral movements are shown respectively (Figure 2B). Next, to investigate the cumulative performance contribution of previous time points for optimal feature construction, all frequency bands were concatenated while continuously increasing the cumulative number of premovement time-points (from -100 to $-1000 \mathrm{~ms}$ ) and each set trained as a Wiener Filter. Note, that this varied the time-point dimension (8 frequency bands $x 10-1$ concatenated time points). The respective best channel $R^{2}$ performances are shown in Figure 2C. A performance saturation becomes visible when concatenating 5 time-points from $500 \mathrm{~ms}$ (prior to target sample) to 0 $\mathrm{ms}$ (target sample), resulting in an optimal input vector of 8 frequency bands with 5 time-points $(=40$ features) for further analyses.

\section{XGBOOST regression shows highest decoding performance for grip-force prediction}

In order to build a grip-force decoder, different machine learning (ML) algorithms were tested in a largescale Bayesian Optimization hyperparameter search. Elastic-net regularized Linear Models, Neural Networks and Gradient Boosted trees (XGBOOST ) (Chen and Guestrin, 2016) were tested for each channel for contra- and ipsilateral movements. To further utilize potential information derived from spatial patterns, the Source Power Comodulation (SPoC) framework (Dähne et al., 2014) was used in combination with elastic-net or XBGOOST predictors. As mentioned, each model was informed by 40 features ( 8 specific frequency bands concatenated at 5 time-points ranging from $t=-500 \mathrm{~ms}$ to $t=0 \mathrm{~ms}$ to movement onset) per channel. For at least 10 Bayesian optimization rounds new hyperparameter settings were tested. Each setting was validated using a nested inner 3-fold Cross-Validation. The objective function metric was the mean of the resulting test set prediction using the $R^{2}$ coefficient of determination. The resulting performances were obtained from the mean left out test set prediction from the outer 3-fold cross validation. This allowed efficient hyperparameter optimization without circularity. 
For statistical testing we conducted permutation statistics that are free from assumptions on data distribution (Modarres and Good, 1995).

Figure 3 shows performance outcomes for the different machine learning methods, with overall best results achieved by XGBOOST. As expected, decoding performances from contralateral ECoG strips show statistical significance over ipsilateral ones (contralateral $R^{2}=0.31 \pm 24$, ipsilateral $R^{2}=0.13 \pm 0.16$, $p$ value=0.02). ECoG XGBOOST performances showed higher performance differences to other $M L$ methods for contralateral movements (Table S2). Thus, ECoG performances increased as long as the complexity of the machine learning algorithms increased, while STN performances saturated regardless of model complexity.

\section{Decoding performance is not improved by combining ECoG and STN signals}

The machine learning methods comparison revealed that XGBOOST performed best across recording modalities. Therefore, we extended our analysis on this approach to accommodate multichannel feature inputs, including combinations of the best pair of ECoG and STN channels (contralateral $R^{2}=0.23 \pm 0.25$; ipsilateral $R^{2}=0.16 \pm 0.21$ ), all ECoG channels available for each hemisphere (contralateral $R^{2}=$ $0.22 \pm 0.22$; ipsilateral $R^{2}=0.12 \pm 0.16$ ), all STN channels available for each subject (contralateral $R^{2}=$ $0.06 \pm 0.15$; ipsilateral $R^{2}=0.04 \pm 0.07$ ). Again, a significant performance advantage of ECoG was observed when compared to STN-LFP (Figure 4A), without additional advantages through higher channel counts or combination of all ECoG and STN channels in a single XGBOOST model (contralateral $R^{2}=0.23 \pm 0.22$; ipsilateral $R^{2}=0.14 \pm 0.18$ ) (Figure $4 \mathrm{~B}$ ).

\section{Decoding performance is correlated to hypodopaminergic state}

To investigate a potential interaction of decoding performance and level of hypodopaminergic state in Parkinson's disease, we calculated Spearman's correlations between preoperative OFF medication UPDRS-III total scores and decoding performance using the all-channel XGBOOST model. Total UPDRS-III scores showed negative correlation to the patient wise mean of contra -and ipsilateral performances for ECoG $(\rho=-0.55, p$-value=0.0426) and STN $(\rho=-0.55, p$-value $=0.039)$ channels (Figure 5A+B). Combined ECoG and STN channel performance also showed significant correlation $(\rho=$ -0.54, p-value $=0.0451)$, as well as combined ECoG $(\rho=-0.55$, p-value $=0.0453)$ and combined STN $(\rho=$ -0.61, $p$-value=0.0236). This correlation was temporally specific to decoding of ongoing grip force, indicative of the models' underestimation of motor output. This suggests that during hypodopaminergic parkinsonian states, the kinematic encoding capacity of the motor system (i.e. both STN and cortex) may be reduced (since less variance can be decoded; Figure $5 \mathrm{C}$ ). 


\section{Spatial distribution of grip-force decoding performance in cortical and subthalamic recordings}

The spatial distributions of decoding performance on cortex and STN for contra- and ipsilateral movements are shown in Figure 6. To evaluate the relevance of recording location with respect to decoding performance, we calculated correlations of performance measures with a priori defined implantation targets. For the subthalamic nucleus this was the dorsolateral STN, corresponding to optimal stimulation site as described in (MNI coordinates: $x= \pm 12.6, y=-13.4 ; z=-5.9$ Caire et al., 2013; Horn et al., 2017a). For the ECoG strip, this was the hand knob area of motor cortex (MNI coordinates: $x= \pm 37, y=-25, z=62$; Mayka et al., 2006). Linear mixed effects models were fit to investigate the relationship between spatial location of recording sites and decoding performances within subjects from contralateral electrodes. The distances between recording sites and hand knob or dorsolateral STN location (see above) were introduced as fixed effects with a random subject effect to allow for varying number of channels and locations across subjects. The models showed a significant relation for contralateral ECoG decoding performances $(\beta=-0.002$, Lower $\mathrm{Cl}=-0.003$, upper $\mathrm{Cl}=-0.001$, $R 2=0.57, p$-value $<0.001)$, but not STN locations $(p>0.05)$. Modelling random effects was only possible post-hoc and thus cannot be used to predict performance across channels and patients in a crossvalidated manner as usually conducted for machine learning. We therefore assessed whether individual channel distances could predict decoding performances across subjects without adding the random effect. Here, we found that the $R^{2}$ performance could not be significantly explained by the ECoG distance to the hand knob area or the STN contact distance to the sensorimotor STN target (leave one channel out prediction ECoG CON p-value $=0.27$, STN CON p-value $=0.13$; leave one patient out prediction ECoG CON p-value $=1$, STN CON p-value $=0.45)$. Thus, Euclidean distance hand knob area for ECoG and therapeutic target for STN was significantly correlated with decoding performance, but could not predict decoding performance across channels or patients.

\section{Whole brain connectomics can be used to cross-predict decoding performances across patients} Being able to account for decoding performances for invasive electrodes may soon become be as important as accounting for variance in therapeutic effects, as bidirectional clinical brain computer interfaces will rely both on electrical sensing and stimulation. Recently, network mapping of neurostimulation effects has shown utility to predict variance in clinical outcomes following DBS (Horn and Fox, 2020; Horn et al., 2017b; Li et al., 2020). Here, we extended the same framework to instead predict variance in grip-force decoding performance observed from single channels, using the XGBOOST grip-force decoding results. In this approach - termed prediction network mapping - we calculated functional and structural connectivity fingerprints by projecting each recording location to a group connectome that was acquired in a cohort of PD patients. These fingerprints denote to which 
other brain areas each site is connected to. First, we tested potential statistical family wise error corrected voxel-wise correlations of connectivity with decoding performance using cluster family-wise error corrected voxel-wise mixed effects models. Functional connectivity strengths between recording sites and sensorimotor cortex (peak coordinate $x=-38, y=-22, z=72)$, parietal lobe $(x=6, y=-32, z=$ 82), striatum $(x=-34, y=-24, z=26)$ and cerebellum $(x=18, y=-50, z=-50$ and $x=-22, y=-52, z=-$ $54)$ accounted for decoding performance. Similarly, for structural connectivity, a significant cluster in the sensorimotor region $(x=-44, y=-18, z=70)$ correlated with high decoding performance. Again, modelling of random effects is not suitable for out-of-cohort prediction. Therefore, we followed two separate approaches that had previously been developed to predict therapeutic DBS effects (see methods section). First, using a discriminative fiber tracking analysis (Baldermann et al., 2019; Li et al., 2020), we analyzed the predictive value of structural connectivity from ECoG recording locations (for an exemplar case see Figure 7A) for XGBOOST decoding performance. Therefore, false discovery rate ( $\alpha$ $=0.05$ ) thresholded $t$-scores were assigned to tracts traversing to more than $20 \%$ of recording locations (Figure 7B). The specific fiber distributions could predict decoding performance of left out channels $(\rho=$ $0.38, p$-value $<0.0001)$ and patients $(\rho=0.37$, $p$-value $<0.0001)$ in a cross validated manner (Figure 7 D).

Next, we created spatial models of optimal decoding performance for functional connectivity (R-Maps are shown in Figure $7 \mathrm{C}$ ). This model led to significant predictions of decoding performance in leaveone-channel -out $(\rho=0.37, \mathrm{p}$-value $<0.0001)$ and leave-one-subject-out cross validations (functional connectivity $\rho=0.37$, $\mathrm{p}$-value $<0.0001$ ) cross-validation (Figure $7 \mathrm{E}$ ). Models such as the two presented here could be used to identify optimal networks that may be used to optimally decode behavior specific brain signals.

\section{Discussion}

Bidirectional brain computer interfaces will revolutionize the treatment of previously intractable brain disorders with brain signal decoding based adaptive neuromodulation. Deep brain stimulation (DBS) provides a unique platform to trailblaze neurophysiological approaches, disease specific modulation and computational strategies for next-generation brain implants improving PD symptoms. Here, we investigated clinical and computational strategies for grip-force decoding based on multimodal invasive neurophysiology in PD patients undergoing DBS neurosurgery. Our findings can be broken down into four major advances to the field: 1) we developed a new decoding approach based on multispectral time-concatenated frequency bands, subjected to Bayesian optimized extreme gradient boosted ensembles (XGBOOST): this outperformed traditional linear model-based methods and may be 
generalized to all brain signal-based regression problems. 2) Next, we demonstrate that electrocorticography signals outperform subthalamic LFP for movement decoding, supporting the utility of additional ECoG in adaptive DBS research. 3) Our findings link loss of coding capacity with high symptom severity/loss of dopamine in PD patients. These highlights higher order interactions of brain signal decoding performance with pathological brain states. Thus, the effect of disease related changes in machine learning performance must be studied and characterized to optimize therapeutic utility of smart implants. 4) Finally, we could significantly predict how well a specific recording site would perform to decode grip force based on brain connectivity. This novel framework (termed prediction network mapping) will be used in future implants to identify connectomic brain networks from which brain sensing can predict symptoms and behavior.

\section{Limitations}

Our analysis is retrospective in nature. Therefore, all model training and evaluations were conducted offline. Nevertheless, all feature extraction and normalization steps are obtained in a pipeline that would be compatible with real-time applications and we took meticulous care to exclude any circularity in processing and machine learning applications. To this date, such circularities are overlooked in some movement decoding papers with filtering, normalization and time frequency transformation across entire sessions, thus reaching into the future from the point of the individually decoded sample. Ridding our analysis from data that would be unavailable in a real-time setting as reported in this study, leads to worse performances, but gives a more realistic estimate of model performance in the clinical use-case. An additional limitation was the relatively small amount of available data, which was constrained by the intraoperative setting (see table 1). For deep learning approaches we hypothesize better performances with an increased dataset size, which in the future will be obtained through longer term recordings, either with externalized leads (He et al., 2020) or sensing enabled implantable devices (Gilron et al., 2020; Opri et al., 2020a).

\section{Decoding grip force based on invasive electrophysiology}

Our study defines a novel computational strategy to decode movement kinematics based on ECoG and LFP in patients undergoing DBS for Parkinson's disease. It explores defined oscillatory feature sets and compares machine learning models with varying complexity, from linear models to artificial neural networks and regression trees. ECoG based movement decoding of varying movement types was previously reported in epilepsy patients that underwent electrophysiological monitoring (Leuthardt et al., 2004) with local motor potential and gamma band activity reported to be informative features (Gunduz et al., 2016). Previous analyses on decoding of grip force based on STN-LFPs was based on Wiener Filter 
architectures (Shah et al., 2018; Tan et al., 2016). A direct comparison of ECoG and LFP and systematic machine learning methods was lacking. Our findings indicate that ECoG recordings are more informative for grip-force decoding than LFP recordings from the STN. Our results are based on extracted band-power features and show superior performances with XGBOOST, when compared to other model architectures and algorithms. The limited recording lengths in our study may be suboptimal for more complex Neural Network architectures that are prone to overfitting. End-to-end learning based on raw signals (Schirrmeister et al., 2018) could be an efficient implementation worth exploring in larger datasets in the future. The small amount of features and efficient automatic feature extraction by in-built regularization lead to highest performance of XGBOOST, that has recently gained attention for $\mathrm{BCI}$ applications (Yao and Shoaran, 2019). Best performances were obtained by single channel ECoG recordings with optimal signal to noise ratio with respect to movement related modulation. We also tested the combination of all ECoG channels, all STN-LFP channels and all ECoG and STN-LFP channels combined, which could not reach the individual best channel performance. Decoding of movement parameters may be a crucial first step in clinical real-time $\mathrm{BCl}$ in movement disorders (He et al., 2020; Opri et al., 2020a) and other motor system diseases such as stroke and spinal cord injury.

\section{Towards machine learning based adaptive stimulation in Parkinson's disease}

Adaptive and responsive neuromodulation has the potential for significant innovation in neuromodulation (Starr, 2018). The beta single- (Arlotti et al., 2018; Little et al., 2013) or dual threshold (Velisar et al., 2019) based is now being investigated in clinical trials to improve the treatment for patients with akinetic rigid dominant Parkinson's disease. Beyond subthalamic beta, ECoG recordings were previously used to successfully indicate the presence of dyskinesia through elevated levels of gamma band synchronization and reduce stimulation intensity (Swann et al., 2018). Single biomarker approaches have the advantage that pathophysiological mechanisms may be the direct target of intervention, while machine learning based decoding methods derive correlates of symptoms and behavior indirectly through learning potentially noisy correlations (Neumann et al., 2019a). Therefore, single biomarker aDBS presents an optimal starting point for investigating the clinical utility of aDBS in controlled study designs. However, single biomarkers alone cannot account for the diverse and complex set of clinical signs of PD and behavior, e.g. during gait, speech and tremor. Here a versatile decoding based control algorithm may further improve clinical outcome for these patients in the future (Neumann et al., 2019b). Indeed, machine learning-based decoding has been successfully described in first translational breakthrough studies. Most notably, the first use of a combined ECoG+LFP based fully embedded machine learning based clinical $\mathrm{BCl}$ approach was reported in three patients with essential tremor who were followed up over more than 6 months (Opri et al., 2020b). Another recent study reports first group 
results in a cohort of $8 \mathrm{ET}$ patients using machine learning based movement and posture decoding for adaptive DBS control (He et al., 2021). Across all reports, machine learning based aDBS showed similar efficacy, compared to conventional continuous stimulation at significantly reduced stimulation ON time (Castaño-Candamil et al., 2020; Ferleger et al., 2020; Houston et al., 2019; Opri et al., 2020b). In Parkinson's disease, recent work has highlighted the utility of combined ECoG+LFP recordings for longer term decoding of bradykinesia and response to medication and neuromodulation in over 500 hours of recording time (Gilron et al., 2020).

In a complementary approach, we focused on direct kinematic decoding, motivated by the intuition that increasing movement vigor and treating bradykinesia with DBS is key for successful treatment in PD, also during movement. Being able to decode movement kinematics can improve aDBS control algorithms for PD and other movement disorders, especially in combination with beta power-based approaches. As shown in this study, subthalamic beta activity is suppressed during movement (also during tremor). While studies indicate that relative amounts of beta can still signal bradykinesia during movement (Feldmann et al., 2021; Lofredi et al., 2019), an adjustment of control parameters would clearly benefit aDBS control, e.g. by keeping stimulation relatively stable or proportional to movement. Machine learning based brain signal decoding may also have diagnostic utility for monitoring motor symptom severity and could inform additional control algorithms to account for bias in traditional biomarkers such as beta and gamma elicited through other voluntary actions (Neumann et al., 2019b). Importantly, we demonstrate that motor symptom severity itself can have direct negative effects on decoding performance, which we should keep in mind during clinical decision making. Previously on a single trial level, the presence of beta bursts correlated with motor performance in cortex (Little et al., 2019) and STN (Torrecillos et al., 2018), which could degrade decoding performance (Khawaldeh et al., 2020). Our findings indicate general impact of motor symptoms in the hypodopaminergic state on machine learning based kinematic decoding capacity. This finding highlights the conceptual relevance of disease specific interactions with computational models. Interestingly, in the hypodopaminergic state, the model output underestimated the grip force extent produced by the patients. It may be speculated that this reflects a loss of neural vigor representations related to insufficient dopaminergic modulation (Turner and Desmurget, 2010). In the future, we will have to account for the individual impact of disease specific changes in brain signals that affect decoding performance. Further, our results support the notion that dopamine plays a key role in coding and modulating neural representations of movement kinematics in the human brain. 


\section{Brain connectivity can help identifying networks with high decoding performance}

In the future, decoding performance for clinical $\mathrm{BCI}$ may be drastically improved when adjusting brain signal recording sites to the underlying interconnected network that is relevant to the specific behavior that is being decoded. For instance, when decoding language or speech, one could picture that recordings at either Broca's or Wernicke's region could be helpful, but a combination of both could be better. The two form a network with direct connections via the Arcuate Fascicle. Based on the data we have which spans across multiple brain regions across the entire cohort, we aimed at identifying the network that would be most useful to decode grip force, as a whole. For this endeavor, we adapted two existing methods that are able to isolate i) connected voxels and ii) connected fibertracts methods (Horn et al., 2017b; Li et al., 2020) associated with a specific effect (such as decoding performance in the present case). While Euclidean distance to motor target, i.e. hand knob area for ECoG and therapeutic target for STN was significantly correlated with decoding performance, this simplistic notion could not predict decoding performance across channels or patients. Given the complexity and vast distribution of movement related brain areas, from cerebellum to frontal cortex to parietal cortex, it may not be surprising that whole brain connectomics outperform single ROI based distance metrics for predicting informative recording locations. Using normative connectivity data allowed us to sample structural and functional wiring diagrams derived from a PD specific dataset. Specific recording locations from intra- or postoperative imaging can be used to identify the underlying whole-brain network architecture, which can then be modelled to predict decoding performance across patients. Such connectomic models can be trained based on multiple dimensions of input-output relationships, e.g. decoding of behavior like grip-force, but also clinical signs, such as tremor. Thus, our results highlight the utility of whole-brain connectomics to predict machine learning-based brain signal decoding performance that can be generalized to any bidirectional clinical brain-computer interface use case. In the future, neurosurgeons may not target individual locations in isolation, but instead determine optimal implant trajectories in accordance with whole-brain connectomic fingerprints for optimal $\mathrm{BCl}$ performance.

\section{Conclusion}

Our analysis from Parkinson's disease patients undergoing DBS implantation showed that ECoG recordings outperform STN-LFP recordings for grip-force decoding throughout different machine learning methods, with XGBOOST showing the highest performance. Parkinsonian motor symptom severity was associated with loss of decoding performance, indicating a specific link of kinematic coding capacity and loss of dopaminergic modulation. Next, we have formalized a connectomic framework that could cross-predict decoding performances across recording sites and patients, based on underlying whole brain MRI connectivity patterns. Our findings highlight the utility of ECoG for intelligent adaptive 
stimulation in Parkinson's disease, corroborate the role of dopamine in kinematic coding and pave the way for connectomic neurosurgery for machine learning-based brain signal decoding.

\section{Acknowledgements}

We would like to thank the patients for participating in the research. The present manuscript was supported through a US-German Collaborative Research in Computational Neuroscience (CRCNS) grant to A.A.K., R.S.T., R.M.R. and W.J.N. with funding from the German Federal Ministry for Research and Education and NIH (R01NS110424). Further funding was provided through Deutsche Forschungsgemeinschaft (DFG, German Research Foundation) - Project-ID 424778381 TRR 295 to A.A.K., A.H. and W.J.N. A.H. was supported by the German Research Foundation (Deutsche Forschungsgemeinschaft, Emmy Noether Stipend 410169619 and 424778381 - TRR 295) as well as Deutsches Zentrum für Luft- und Raumfahrt (DynaSti grant within the EU Joint Programme Neurodegenerative Disease Research, JPND).

Some data used in the preparation of this article were obtained from the PPMI database (www.ppmiinfo.org/ data). For up-to-date information on the study, visit www.ppmi-info.org. PPMI, a public-private partnership, is funded by the Michael J. Fox Foundation for Parkinson's Research. For funding partners, see www.ppmi-info.org/fundingpartners.

\section{Figure Legends}

Figure 1: Movement induced spectral changes are more dominant for ECoG than STN-LFP signals for a grip force task before and after a machine learning feature signal processing pipeline. (A) ECoG, STN and gripping force were recorded simultaneously. (B) Individual ECoG and STN electrodes were localized (and transformed into) in Montreal Neurological Institute (MNI) space. (C) Mean spectral power of all ECoG and STN channels for contra -and ipsilateral movements showed typical movement induced spectral changes. (D) Virtual streaming of data packets secured real-time compatible processing and normalization to extract time-frequency modulations into discrete feature time-series. (E) Schematic flow chart of the implemented real-time enabled feature extraction, machine learning evaluation and functional and structural connectivity analysis pipeline.

Figure 2: Linear Model analysis shows movement typical frequency band coefficients and linear Wiener Filter performance comparison show performance gain of concatenated features with a 
lag up to $\mathbf{5 0 0}$ ms. (A) Best patient specific linear model coefficients show movement induced spectral changes, with stronger deflection for ECoG than STN-LFP signals. (B) Individual frequency band and time shifted Linear Model coefficients reveal movement specific feature importance. HFA and $\mathrm{Y}$ bands show stronger deflection for contralateral over ipsilateral movements. Moreover, stronger deflections are visible for ECoG over STN-LFP signals for $\beta, H F A$ and $\gamma$ bands. (C) Linear Wiener Filters of cumulative time-points demonstrate performance gain of concatenated features from $-500 \mathrm{~ms}$ prior to the target sample up to $0 \mathrm{~ms}$ (target sample of grip-force measure) for ECoG and STN-LFP signals.

Figure 3: XGBOOST regression shows highest movement decoding performance as compared to other machine learning methods. Based on the presented real-time compatible signal processing pipeline Elastic Net regularized Linear Models (LM) and Wiener Filters (WF), Neural Networks (NN) and extreme Gradient Boosting (XGBOOST) regression was tested. Mean $R^{2}$ performances are shown for the best patient specific channel of optimal Bayesian Optimization obtained hyperparameters. The same pipeline was applied for a spatial feature approach using all available channels of an electrode for each patient with Source Power Comodulation (SPoC). Best ECoG performances were obtained by XGBOOST regressors. STN-LFP signals did not exhibit performance gain when applying advanced machine learning methods. The mean ECoG minus STN XGBOOST performance differences of contralateral $\Delta R^{2}=0.21 \pm 0.18$ and ipsilateral $\Delta R^{2}=0.069 \pm 0.08$ movements, indicate the higher gripforce decoding performance of ECoG over STN signals. Throughout every patient the mean test set prediction performances were higher for ECoG than for STN, for both contra -and ipsilateral movements.

Figure 4: ECoG decoding performance significantly outperforms STN-LFP decoding performance. (A) Individual best XGBOOST decoding performances show throughout every patient performance gain of ECoG over STN-LFPs (contralateral p-value=0.0007, ipsilateral p-value=0.035). (B) When combining multiple channels, best performances are achieved with combined ECoG and STN but performance remains below individual best ECoG as depicted in (A). (C) Exemplary test set of best individual ECoG contralateral prediction and ground truth grip-force trace.

\section{Figure 5: XGBOOST $R^{2}$ performances show negative PD UPDRS-III clinical symptom severity} correlation. UPDRS-III scores show significant negative correlation to mean contra- and ipsilateral channels for (A) ECoG $(\rho=-0.55, p$-value=0.0426) and $(B)$ STN performances $(\rho=-0.55, p$ value $=0.039$ ). $(C)$ The temporal specificity of this correlation is revealed through movement aligned sample-wise correlations of average force predictions with UPDRS-III scores across patients (cluster based corrected significant segments are displayed shaded). 
Figure 6: Mean test XGBOOST $R^{2}$ performances peak around cortical sensorimotor areas and the dorsolateral STN. (A) Channels are color coded for individual XGBOOST regression performances based on the best Bayesian Optimization hyperparameter set. Performance differences shown are in favor of ECoG over STN and contralateral over ipsilateral recording locations for movement decoding. (B) Interpolated cortex performance peaks in sensorimotor areas. STN interpolated decoding performance peaks at the dorsolateral STN which was shown to overlay with the optimal PD DBS target (Caire et al., 2013).

Figure 7: Structural and function movement decoding network analysis reveals cerebellar as well as sensorimotor cortical decoding capacity. (A) Visualization of fibers originating from the ECoG recording locations of subject 1. (B) Leave-nothing-out decoding performance significant $(\alpha=0.05$ FDR corrected) fiber tracts are shown across subjects. (C) The optimal R-Map is shown for the cortical surface as well as cerebellum for fMRI functional connectivity. (D) Fiber tracking connectivity predicts decoding performance (leave one channel out cross validation $\rho=0.38, p<0.0001$, leave one patient out cross validation $\rho=0.24$, $p$-value $=0.0004)$. (E) Functional connectivity predicts decoding performance (leave one channel out cross validation $\rho=0.37, p<0.0001$, leave one patient out cross validation $\rho=0.25, p=0.0004)$.

\section{Author Contributions}

Conceptualization, T.M., R.M.R. \& W.J.N.;

Methodology, T.M., V.P., W.L., A.H., R.M.R., W.J.N.;

Software, T.M., V.P., A.H., N.L., W.J.N.;

Validation, W.J.N.;

Formal Analysis, T.M. \& V.P.;

Investigation, W.L., R.M.R.;

Resources, A.H., A.A.K., R.M.R., W.J.N. ;

Data Curation, R.M.R.;

Writing - Original Draft, T.M.;

Writing - Reviewing \& Editing, V.P., W.L., A.H., B.B., T.M., A.A.K., R.S.T., R.M.R., W.J.N.;

Visualization, T.M., W.J.N;

Supervision, B.B., A.A.K., R.S.T., R.M.R., W.J.N.;

Project Administration, A.A.K., R.M.R., W.J.N.;

Funding Acquisition, B.B., T.M., A.A.K., R.S.T., W.J.N, M.R. 


\section{Declaration of interests}

The authors declare no competing interests.

\section{Lead contact}

Further information and requests for resources should be directed to and will be fulfilled by the Lead Contact, Wolf-Julian Neumann (julian.neumann@charite.de).

\section{Materials Availability}

The code for the reproduction of every Figure, machine learning and statistical analysis are publicly available at the GitHub repository (https://github.com/neuromodulation/icn/tree/master/ECoG vs STN).

\section{Data and Code Availability}

The code is open-source and can be downloaded from GitHub repository (https://github.com/neuromodulation/icn/tree/master/ECOG vs STN).

\section{Participants}

The current study is based on previously published data (Alhourani et al., 2020). In brief, subthalamic LFP and subdural ECoG recordings were simultaneously acquired from 11 PD patients. The patients were subjected to bilateral STN DBS lead implantation, as proposed by standard clinical indications criteria. In accordance with protocol \#PRO13110420, approved by the Institutional Review Board of the University of Pittsburgh, informed consent for all patients was obtained prior to any surgical procedure. The subject characteristics are detailed in Table 1. UPDRS Part III scores for the off-medication conditions were collected in a time period of 1-3 months prior to surgery by movement disorder neurologists. Antiparkinsonian medications were held for at least 12 hours before intraoperative testing.

\begin{tabular}{|c|c|c|c|c|c|c|}
\hline $\mathrm{N}$ & Gender & $\begin{array}{c}\text { UPDRS } \\
\text { total }\end{array}$ & $\begin{array}{c}\text { Hemisp } \\
\text { here }\end{array}$ & Age & Movements & $\begin{array}{c}\text { Disease } \\
\text { duration } \\
\text { [years] }\end{array}$ \\
\hline 0 & Male & 28 & $\mathrm{R}$ & 60.3 & 128 & 10.7 \\
\hline 1 & Male & 27 & $\mathrm{~L}+\mathrm{R}$ & 51.2 & 464 & 14 \\
\hline 2 & Male & 33 & $\mathrm{~L}+\mathrm{R}$ & 53.8 & 213 & 7.2 \\
\hline 3 & Male & 31 & $\mathrm{~L}+\mathrm{R}$ & 44.2 & 285 & 10.1 \\
\hline 4 & Male & 32 & $2 \mathrm{~L}+2 \mathrm{R}$ & 63.6 & 381 & 13.1 \\
\hline 5 & Male & 52 & $\mathrm{~L}$ & 59.6 & 84 & 5.9 \\
\hline
\end{tabular}




\begin{tabular}{|c|c|c|c|c|c|c|}
\hline 6 & Male & 55 & $\mathrm{~L}$ & 71.6 & 161 & 1.4 \\
\hline 7 & Male & 50 & $\mathrm{~L}$ & 52.5 & 131 & 8.7 \\
\hline 8 & Male & 62 & $\mathrm{~L}+\mathrm{R}$ & 66.8 & 547 & 9.8 \\
\hline 9 & Male & 48 & $\mathrm{~L}$ & 67.9 & 86 & 17.1 \\
\hline 10 & Female & 31 & $\mathrm{R}$ & 69 & 205 & 10.4 \\
\hline
\end{tabular}

Table 1: Subject characteristics

\section{Behavioral Paradigm}

The behavioral task performed for this study was previously described (Alhourani et al., 2020; Fischer et al., 2020; Kondylis et al., 2016) and it is schematically shown in Figure 1A. For this, the subjects were first submitted to DBS lead implantation. Subjects were fully awake, and no anesthetic agents were administered for at least 1 hour before the task procedure. No medication was given during the task.

The task paradigm was implemented using the Psychophysics Toolbox (Brainard, 1997) on a portable computer. The trials consisted of a simultaneous presentation of a yellow traffic light in the center of a screen, and a cue on one side indicating which hand the subject should use for the subsequent response of squeezing the handgrip. The cue remained on screen for 1000-2000 ms, followed by the traffic light changing either green or red, signaling a "go cue" and "no-go cue" respectively. Subjects performed the task for a cumulative total time of 10 to $25 \mathrm{~min}$. As the present study focuses on force and movement decoding performance based on the electrophysiological signals, all sessions containing valid movements were merged per subject for further analysis.

\section{Electrophysiological Recordings}

ECoG data were recorded intra-operatively from subjects with Parkinson's disease using six-contact (left $n=5$ patients, right $n=3$ ), eight-contact (left $n=3$, right $n=3$ ) and twenty-eight-contact (left $n=2$, right $n=2)$ strip electrodes. The electrode details are shown in Supplementary Table 1 and all ECoG and STN electrodes are plotted in Figure 1B. Temporarily implanted ECoG recording strip contact number varied (left, right, some both), mean electrode contacts were left 10.18士11.29 and right: 8.9土12. A referential montage was used in which the reference electrode was placed in the scalp and a ground electrode was placed in the skin overlying the acromion process. LFP data from the lead were obtained after clinical stimulation testing was completed. ECoG and STN signals were filtered $(0.3-7.5 \mathrm{kHz})$, amplified, and digitized at $30 \mathrm{kHz}$ using a Grapevine neural interface processor (Ripple Inc.). Force signals were digitally recorded simultaneously with the ECoG and STN-LFP signals. LFPs from the STN 
were recorded using the clinical DBS lead (model 3389, Medtronic) from all four contacts and referenced offline in a bipolar montage. All signals were resampled to $1 \mathrm{kHz}$ for offline analysis.

\section{Electrode Localization}

Subdural electrode strips were implanted temporarily through standard frontal burr holes located near the coronal suture and aimed posteriorly to the hand knob motor cortex region. Strip targeting has been previously described and was based on markings of stereotactically defined overlying scalp locations (Kondylis et al., 2016). Subdural electrode reconstructions were obtained by aligning pre-operative MRI, intra-operative fluoroscopy, and postoperative CT. Representative images of this technique were previously explained in detail (Randazzo et al., 2016). In short, the CT and MRI were co-registered using mutual information using the SPM software library and rendered onto 3D skull and brain surfaces using Osirix (v7.5) (Rosset et al., 2004) and Freesurfer (v5.3) software packages (Dale et al., 1999), respectively. These surfaces and the fluoroscopy images were then aligned according to common points: stereotactic frame pins, implanted depth electrodes, and skull outline positions (Randazzo et al., 2016). The parallax effect of the fluoroscopic images was accounted for using the obtained distance from the radiation source to the subject's skull. Succeeding the surface-to-fluoroscopic image orientation alignment, a 3D location for each electrode contact was projected from the fluoroscopic image to the cortical surface. Deep brain stimulation electrode locations were reconstructed using the advanced neuroimaging pipeline defined by Lead-DBS using default settings (Horn et al., 2019). In brief, preoperative MRI and postoperative CT scans were co-registered and normalized to MNI 2009b NLIN ASYM space. Electrode artefacts were visually identified and marked to obtain MNI coordinates of DBS electrode contacts. All electrode localizations are visualized in Figure $1 \mathrm{~B}$.

\section{ECOG and LFP preprocessing and feature extraction}

Our results primarily aim to inform the development of brain computer interface applications. Therefore, the preprocessing pipeline used in the present study was optimized for real-time performance and inspired by the Berlin Brain Computer Interface (Blankertz et al., 2006). Processing was performed in Python using custom code based on MNE-python (Gramfort et al., 2013), mne_bids (Appelhoff et al., 2019), fooof (Haller et al., 2018) and pybv (https://pybv.readthedocs.io/en/stable/). All raw data files were saved in the iEEG-BIDS structure (Holdgraf et al., 2019). To account for baseline drifts the force traces were cleaned using a normalization approach presented for previous ECoG finger trajectory decoding (Xie et al., 2018). A real-time data stream of untouched electrophysiological raw data was emulated to ensure that all processing that can impact decoding is performed in a real-time compatible manner. Referencing was performed online, where all LFP recordings were referenced bipolarly, against 
the adjacent contacts (0-1, 1-2, 2-3 with contact 0 being the lowest by convention of the manufacturer). ECoG recordings were referenced by subtracting the common average of all ECoG electrodes. To adhere to most computationally efficient real-time enabled algorithms, time frequency decomposition for the machine learning analysis was conducted by bandpass filtering in the $\theta(4-8 \mathrm{~Hz}), \alpha(8-12 \mathrm{~Hz}), \beta(13-$ $35 \mathrm{~Hz})$, low $\beta(13-20 \mathrm{~Hz})$, high $\beta(20-35 \mathrm{~Hz})$, all $\gamma(60-200 \mathrm{~Hz}), \gamma(60-80 \mathrm{~Hz})$ and high-frequency activity, (90-200 Hz) frequency bands. Overlapping broad $\beta$ and $\gamma$ bands were added in addition to subbands to enable the investigation of distinct interactions within these frequency bands (Figure 1C). In order to estimate band specific activity, different time durations were used for band pass filtering. In this manner longer time segments were used lower frequencies, and shorter time segments for higher frequencies $(\theta$ $=1000 \mathrm{~ms}, \alpha$ and $\beta$ bands $=500 \mathrm{~ms}, \gamma=100 \mathrm{~ms}$ ). Note that, only past samples of the stream could be used for every sample again, compatible with a real-time recording. To get an estimate of amplitude of the activity in the filtered signals, variance was extracted in intervals of $1 \mathrm{~s}$ in a sliding window of $100 \mathrm{~ms}$ resulting in a time resolution of $10 \mathrm{~Hz}$. All variance estimates were normalized by subtracting and dividing by the median in a sliding window of $10 \mathrm{~s}$ to account for differences in impedance and proximity to the source before subjecting the data to the machine learning analysis. By a fixed parameter of -2 and 2 after normalization, all features were clipped as an artifact rejection mechanism. The used normalization is based on the real time prediction approach, such that data acquired in the future do not influence the present predictions. See figure $1 \mathrm{E}$ for an outline of the methods pipeline. For the purpose of visualization, Morlet wavelets $(7$ cycles) were used to demonstrate the entire time-frequency decomposition (Figure 1C).

\section{Machine learning training end evaluation}

In order to model real-time movement decoding, a rigorous nested cross validation approach was implemented. An outer 3-fold cross validation split the data into folds of two third training and one third test set non shuffled. For each individual channel a Bayesian Optimization hyperparameter search (Frazier, 2018) (further details below) was then conducted for at least 10 rounds using the training set only. For each round the training data was trained and tested in an inner non shuffled 3-fold crossvalidation with 80 percent training size. The mean $R^{2}$ coefficient of determination of every test set estimate of the outer cross-validation was used as the performance measure. $R^{2}$ values were Fishertransformed through the inverse hyperbolic tangent function to approximate a normal distribution for further statistics (Fisher, 1915). The input features for every model were all eight previously described frequency bands. In order to test the contribution of previous time points, frequency band features of different time points were concatenated and compared according to their decoding performance. 
The present study investigated commonly used and promising machine learning algorithms, by fitting functions using electrophysiology as input and grip force as the target variable. The chosen methodology is non-exhaustive and primarily serves the comparison of variance in decoding explained by the source of the signal (ECoG vs. STN) and motor symptom severity (UPDRS-III). It further gives an intuition about the potential of more complex and elaborate machine learning methods for brain computer interfaces.

\section{Linear Models}

Linear models can capture underlying feature dependencies and reveal those as correlations in each weight parameter. Input features are multiplied by an according weight coefficient. The dot product of the weight vector $\boldsymbol{w}$ and feature vector $\boldsymbol{x}$ is then shifted by the bias $b$. The feature vector in this analysis is the vector of all frequency bands for a single time point. The prediction label $y$ is the baseline corrected gripping force. For a linear regression the activation function is linear, is defined as follows:

$$
y=\boldsymbol{w} \boldsymbol{x}+b
$$

For classification, a saturating transfer function is used (e.g. hyperbolic tangent or sigmoidal). To prevent overfitting, regularization in the form of $l_{1}$ and $l_{2}$ norm is commonly used. Here we tested different parameters of the elastic-net (enet) regularization (Zou and Hastie, 2005), which is a combination of the $l_{1}$ and $l_{2}$ norm specified by the regularization hyperparameters $\alpha$ and $\rho$, respectively. The objective function of the enet model follows:

$$
\min _{w} \frac{1}{2 n_{\text {samples }}}\|\boldsymbol{X} \boldsymbol{w}-\boldsymbol{y}\|_{2}^{2}+\alpha \rho\|\boldsymbol{w}\|_{1}+\frac{\alpha(1-p)}{2}\|\boldsymbol{w}\|_{2}^{2}
$$

where $\boldsymbol{X}$ is a matrix of dimension $n \times m$ whom $\mathrm{i}^{\text {th }}$ row is the feature vector $\boldsymbol{x}$ of size $m$ and $\boldsymbol{w}$ is the solution vector, which, due to the $l_{1}$ sparse regularization term, most of the coefficient will be expected to be zero. For hyperparameter-search, $\alpha$ and $\rho$ were both sampled from a uniform distribution ranging from zero to one. Since elastic nets are solved using gradient descent, the maximum training iteration also needs to be specified. Here an iteration of 1000 has been used. The implementation was done using the scikit learn Python package (Pedregosa et al., 2011).

\section{Wiener Filtering}

Tan et al. described the use Wiener filters in the application of force estimation from STN-LFP signals (Shah et al., 2018). Here the output $y$ is a weighted sum of features in the time and frequency domain in the weight matrix $\boldsymbol{W}$. I frequency band features are used together with $J$ lags. For the regression analysis the activation function is kept linear, as follows: 


$$
y(n)=\sum_{j=0}^{J} \sum_{i=0}^{I} w_{i j} x_{i}(n-j)
$$

This equation has a closed form solution, known as the normal equation (Proakis and Monolakis, 1996). Wiener filters essentially implement a multivariable linear model with multiple time-steps. Using Wiener filters we tested the contribution of different concatenated time parameters. This gives insight about the optimal feature length in the time domain.

\section{Neural Networks}

We have further investigated the utility of artificial neural networks. While linear models and Wiener filters may underfit the data, neural networks can be very complex and have a higher risk to overfit with increasing complexity. The ideal model architecture finds a balance between under and over- fitting to the training dataset. In this context not only single weight correlations of band features could contribute to force decoding performances, but a richer representation of feature invariances in combinations of different frequency bands may be learned by additional layers and units of the model. The architecture of neural networks is derived from linear models with non-linear activation functions, which are referred to in this context as units. Multiple units are combined in different layers with different activation functions.

Explicitly, the output $y$ of the $\mathrm{i}^{\text {th }}$ unit in layer $l$ is the weighted sum of activations of the previous layer units $y_{k}^{l-1}$ with weights $w_{i k}^{l}$,

$$
y_{i}^{l}=f^{l}\left(\sum_{k} w_{i k}^{l} y_{k}^{l-1}+b_{i}^{l}\right)
$$

Neural networks are trained through a cost function using a gradient descent algorithm. Hyperparameters were adjusted in order to prevent over -and underfitting (Geman et al., 1992). Here neural networks were tested with at least one hidden layer. The input nodes of this layer were in the hyperparameter search uniformly sampled in a range of 1 to 10 . The number of hidden dense layers were sampled from a range of 1 to 3 layers. The hidden dense layer neurons were uniformly sampled in a range of 1 to 10. Sigmoidal and hyperbolic tangent activation functions were tested in the hidden layers. After each hidden layer a batch normalization layer and a d dropout layer with a factor of 0.2 was added. The output activation function was set linear. The used training algorithm was the Adam optimizer (the learning rate was sampled from a log uniform distribution from 0.0001 to $0.01, \beta_{1}$ was set to $0.9, \beta_{2}$ to 0.999 and $\varepsilon$ to 0.999 ). The Adam optimizer improves backpropagation such that each weight parameter is adapted according to its first and second momentum (Kingma and Ba, 2015). Each 
neural network was trained using 1000 epochs with a batch size of 100 . The loss function was set to the mean squared error. To prevent overfitting, the training set was further split into train and validation set with 80 percent train. The validation dataset was then used for early stopping with a patience parameter of 10 epochs. The model with lowest validation error is then used for test set prediction. Due to poor performances, the inner cross validation was left out for the neural network training sequence. Neural Networks were implemented using the TensorFlow framework (Abadi et al., 2016).

\section{Gradient Boosted Trees using the XGBOOST Framework}

A common problem with neural networks is the high dependency on the provided set of features and potential to learn spurious input-output associations. In this analysis a feature vector of all 8 frequency bands concatenated for 5 time points requires a Wiener Filter with 40 weights. In an architecture like neural networks all these features are contributing to the overall force prediction, nevertheless not all weight parameters are promising. Decision Tree algorithms overcome this problem naturally by implementing optimization of input feature use in their architecture. Thus, decision trees and random forests, first described by Breiman (Breiman, 2001), were proven to be a robust, accurate and successful tool for solving machine learning tasks, including classification, regression, density estimation and manifold learning or semi-supervised learning (Gall and Lempitsky, 2013). Random forests are an ensemble method consisting of many decision trees. A decision tree is a statistical optimal data segregation method, that is only controlled by conditional sequences. Different implementations were proposed on top of Decision Trees. AdaBoost (Schapire, 2009) is an adaptive learning algorithm that builds up successive decision trees iteratively. By that an ensemble of multiple weighted weak learners are combined to yield a strong estimator. Gradient Boosting is built using the same concept. According to Empirical Risk Minimization it fits each decision tree based on the residuals of a defined objective function. This objective function is typically based on an error loss and a regularization term. The model is initialized using a constant value. In an iterative process the new trees are added to the model up till the maximum defined estimators are reached. Here the scalable tree boosting framework XGBOOST (Chen and Guestrin, 2016) was used. In this analysis the number of boosting rounds is set to 10 . The depth of each tree is sampled uniformly in a range from 1 to 100 . When adding new trees to the model the parameter learning rate $\eta$ is scaling the contribution of each tree prediction and is sampled here log uniformly from of the range $\left[10^{-5}, 1\right]$. Regularization in Gradient Boosted Trees is controlled by different factors. One of the factors is the minimum splitting loss $\gamma$. For every decision tree new nodes were added only if the gain metric was above $\gamma$. It is here sampled from a uniform distribution between 1 and 10. Hyperparameters for all used machine learning methods are listed in detail in supplementary table 1. 


\section{Source Power Comodulation}

A state of the art movement prediction approach is the source separating framework called Source Power Comodulation (SPoC) (Dähne et al., 2014). Oscillatory sources are here extracted based on their power comodulation with the force gripping target. SPoC was implemented using the MNE framework (Gramfort et al., 2013). Thus, discriminant neural sources are made visible. In this context, the bandpower at each frequency band of interest was calculated by taking the logarithm of the variance of the projected signal in the source space. For sake of comparison, only one spatial filter was used for feature computation at each frequency band. In the same manner as before, a Wiener filter was then applied in order to resample time lags up to $500 \mathrm{~ms}$. Here again, the band power features are then used as input features. A Bayesian Optimization hyperparameter search was also here implemented for both the enet model as well as the XGBOOST framework with the aforementioned parameters.

\section{Hyperparameter Search: Bayesian Optimization}

A common problem using machine learning algorithms is finding the optimal hyperparameter settings given a certain architecture. Grid search exhaustively tries out all provided hyperparameters while Random search only draws random parameters from the given hyperparameter distributions. Sampling the error loss function can be computationally expensive, given the dataset size. Bayesian Optimization formulates this problem into an optimization problem. Here a cost function should be minimized given a set of hyperparameters. Instead of sampling from the objective cost function, a probabilistic model is stated. The hyperparameters minimizing the negative expected improvement given a multinomial Gaussian process are drawn. Those parameters are then used to sample from the respective regressor in the given dataset. The resulting error updates then the gaussian process distribution and given the maximum expected improvement, the next best hyperparameter set is drawn. This process is repeated for the elastic net and XGBOOST architecture for 20 iterations, for neural networks for 10 iterations due to computational cost of neural networks. For every round a 3 fold cross validation is used in order to prevent overfitting. Given log-uniform distributions a wide range of hyperparameters can thus be sampled in a computational feasible manner. The implementation was done using the scikit-optimize framework (https://scikit-optimize.github.io/stable/).

\section{Definition of best model and best channels}

Previous studies have repeatedly demonstrated that using a single optimal channel in the STN is advantageous over using all available channels (Shah et al., 2018). Most importantly, addition of more channels leads to decreased generalization and higher risk of overfitting with little performance benefit in 
all models, except for XGBOOST. Based on these results and in order to account for varying numbers of available electrode contacts, one channel with optimal decoding performance on the cross-validation test set was chosen per patient to quantify and compare decoding performance for the ECoG and STN analysis across patients.

\section{Prediction Network Mapping with whole-brain connectomics}

Currently, most decoding approaches for brain signals depend on individual patient training. Here, differences in electrode location and underlying neural populations can challenge generalization across patients. To investigate whether decoding performance from different recording locations can crosspredict across patients, we developed a whole-brain connectomics based approach to capitalize on information derived from circuit fingerprints. Therefore, ECoG electrode recording locations were projected to normative structural and functional (Parkinson's Progression Markers Initiative [PPMI]; www.ppmi-info.org) using Lead-DBS software in Matlab (www.lead-dbs.org, (Horn et al., 2019). The PPMI connectomes of patients with PD ( $\square=\square 74$ ) was priorly computed (Ewert et al., 2018) and has been used in context of DBS multiple times (de Almeida Marcelino et al., 2019; Horn et al., 2017c; Lofredi et al., 2020; Neumann et al., 2018). Seeding from each recording site resulted in connectivity profiles (fingerprints) that were expressed as voxel-wise whole-brain volumes for functional and structural connectivity and a set of streamline connections for structural connectivity.

First in a voxel-wise approach, structural connectivity between ECoG channels and all other brain areas was calculated using Lead Mapper (www.lead-dbs.org). In a first statistical voxel-wise correlation between decoding performance and structural and functional connectivity, separate mixed effects models, with a subject based random effect, were corrected for multiple comparisons with random field theory as implemented in the Statistical parametric mapping (SPM12) toolbox (https://www.fil.ion.ucl.ac.uk/spm/). However, this approach is not feasible for out-of-cohort prediction. Therefore, we have adapted two previously published methods leveraging normative connectomes as predictive models. First, structural and functional connectivity maps were used as predictive to generate an "R-Map", a connectivity model which is associated with optimal decoding performance, by performing voxel-wise correlations of connectivity and decoding performance from recording locations. The spatial fingerprint from each recording location can then be assigned a spatial correlation coefficient that may have predictive value for the underlying decoding performance. This predictive value was validated using "leave-one-channel-out" and "leave-one-subject-out" cross-validation. For a left out contact the correlation between the R-Map and the individual fingerprint allows for a decoding performance prediction of that contact. All connectivity analyses were performed using ECoG recording locations with contralateral $R^{2}$ performances (Figure 1E). As a second approach, fiber streamlines representative of 
structural connectivity between ECoG channels and all other brain areas were isolated and assigned with a "Fiber T-score", associating XGBOOST decoding performance with the fiber tracts connectivity from respective ECoG recording locations across patients using mass-univariate two-sample t-tests between $R^{2}$ scores in connected vs. unconnected recording locations. Only fibers with significant tscores surviving FDR correction at an alpha level 0.05 were considered further. Next, T-values were used as weights in an aggregated fiber score to predict out of training sample channel and patients' performances.

\section{Statistics}

Results are stated as mean \pm standard deviation. All significance testing was performed using MonteCarlo permutation and bootstrapping. For paired comparisons, the sign of paired differences is randomly assigned in a surrogate distribution. The $p$-value is assigned by reporting the percentile of the average original paired difference in the surrogate distribution. For correlation analyses, Spearman's correlations were calculated. p-values were obtained by shuffling value positions and determining the resulting original rho value percentile in the distribution of surrogate combinations. Permutations tests are robust to small sample size and do not depend on assumptions with respect to data distribution. Clinical correlations were performed between decoding performances and UPDRS-III total scores. To test for the temporal specificity of the clinical correlation, we performed sample-wise correlations of decoding output with UPDRS-III total scores across subjects. Multiple comparisons were corrected by adjusting the significance threshold $a$ to the false discovery rate (Benjamini and Hochberg, 2000), except where explicitly otherwise stated.

\section{Supplementary video and excel table title and legends}

\begin{tabular}{|l|l|}
\hline Hyperparameters & Elastic Net Regularized Wiener Filter \\
\hline Time lag & $500 \mathrm{~ms}$ \\
\hline L1 ratio alpha & 0 to 1 \\
\hline L1/L2 ratio rho & 0 to 1 \\
\hline iterations & 1000 \\
\hline used library & sci-kit learn \\
\hline & \\
\hline
\end{tabular}




\begin{tabular}{|c|c|}
\hline Hyperparameters & Neural Networks \\
\hline input nodes & 1 to 10 uniform \\
\hline dense layers & 1 to 3 uniform \\
\hline dense neurons & 1 to 10 uniform \\
\hline activation function & sigmoidal and tanh \\
\hline dropout factor & 0.2 \\
\hline Adam learning rate & 0.0001 to 0.01 log uniform \\
\hline Adam beta 1 & 0.9 \\
\hline Adam beta 2 & 0.999 \\
\hline eta & 0.999 \\
\hline batch size & 100 \\
\hline epochs & 1000 \\
\hline loss function & MSE \\
\hline val split & 0.8 \\
\hline early stopping epochs & 10 \\
\hline used library & tensorflow \\
\hline Hyperparameters & XGBOOST \\
\hline boosting rounds & 10 \\
\hline depth & 1 to 10 uniform \\
\hline eta tree scaling & 0.00001 to 1 uniform \\
\hline $\begin{array}{l}\text { splitting loss } \\
\text { regularization }\end{array}$ & 1 to 10 uniform \\
\hline max depth & 6 \\
\hline min child weight & 1 \\
\hline max delta step & 0 \\
\hline sampling method & uniform \\
\hline L1 regularization & 0 \\
\hline $\begin{array}{l}\text { L2 regularization } \\
\text { lambda }\end{array}$ & 1 \\
\hline
\end{tabular}


Table S1 Bayesian Optimization Hyperparameter used, Related to STAR Methods

\begin{tabular}{|l|l|l|l|l|}
\hline ML method & $\begin{array}{l}\text { ECoG } \\
\text { Con. }\end{array}$ & $\begin{array}{l}\text { ECoG } \\
\text { Ips. }\end{array}$ & $\begin{array}{l}\text { STN } \\
\text { Con. }\end{array}$ & $\begin{array}{l}\text { STN } \\
\text { Ips. }\end{array}$ \\
\hline LM & $0.14 \pm 0.1$ & $0.06 \pm 0.04$ & $0.06 \pm 0.11$ & $0.03 \pm 0.03$ \\
\hline Wiener Filter & $0.19 \pm 0.13$ & $0.09 \pm 0.06$ & $0.1 \pm 0.16$ & $0.067 \pm 0.087$ \\
\hline Neural Networks & $0.14 \pm 0.24$ & $0.09 \pm 0.19$ & $0.015 \pm 0.04$ & $0 \pm 0$ \\
\hline SPOC LM & $0.16 \pm 12$ & $0.085 \pm 0.07$ & $0.09 \pm 0.16$ & $0.05 \pm 0.068$ \\
\hline SPOC XGB & $0.23 \pm 0.2$ & $0.09 \pm 0.14$ & $0.09 \pm 0.2$ & $0.05 \pm 0.11$ \\
\hline XGB & $0.31 \pm 24$ & $0.13 \pm 0.16$ & $0.09 \pm 0.2$ & $0.07 \pm 0.1$ \\
\hline
\end{tabular}

Table S2: Best channel mean machine learning $R^{2}$ performance, Related to Figure 3

\section{References}

Abadi, M., Barham, P., Chen, J., Chen, Z., Davis, A., Dean, J., Devin, M., Ghemawat, S., Irving, G., Isard, M., et al. (2016). TensorFlow: A system for large-scale machine learning. Proc. 12th USENIX Symp. Oper. Syst. Des. Implementation, OSDI 2016.

Alhourani, A., Korzeniewska, A., Wozny, T.A., Lipski, W.J., Kondylis, E.D., Ghuman, A.S., Crone, N.E., Crammond, D.J., Turner, R.S., and Richardson, R.M. (2020). Subthalamic Nucleus Activity Influences Sensory and Motor Cortex during Force Transduction. Cereb. Cortex 30, 2615-2626.

de Almeida Marcelino, A.L., Horn, A., Krause, P., Kühn, A.A., and Neumann, W.-J. (2019). Subthalamic neuromodulation improves short-term motor learning in Parkinson's disease. Brain 142, 2198-2206.

Androulidakis, A.G., Kuhn, A.A., Chu Chen, C., Blomstedt, P., Kempf, F., Kupsch, A., Schneider, G.-H., Doyle, L., Dowsey-Limousin, P., Hariz, M.I., et al. (2007). Dopaminergic therapy promotes lateralized motor activity in the subthalamic area in Parkinson's disease. Brain 130, 457-468.

Appelhoff, S., Sanderson, M., Brooks, T., van Vliet, M., Quentin, R., Holdgraf, C., Chaumon, M., Mikulan, E., Tavabi, K., Höchenberger, R., et al. (2019). MNE-BIDS: Organizing electrophysiological data into the BIDS format and facilitating their analysis. J. Open Source Softw.

Arlotti, M., Rosa, M., Marceglia, S., Barbieri, S., and Priori, A. (2016). The adaptive deep brain stimulation challenge. Park. Relat. Disord.

Arlotti, M., Marceglia, S., Foffani, G., Volkmann, J., Lozano, A.M., Moro, E., Cogiamanian, F., Prenassi, 
M., Bocci, T., Cortese, F., et al. (2018). Eight-hours adaptive deep brain stimulation in patients with Parkinson disease. Neurology.

Baldermann, J.C., Melzer, C., Zapf, A., Kohl, S., Timmermann, L., Tittgemeyer, M., Huys, D., VisserVandewalle, V., Kühn, A.A., Horn, A., et al. (2019). Connectivity Profile Predictive of Effective Deep Brain Stimulation in Obsessive-Compulsive Disorder. Biol. Psychiatry.

Benjamini, Y., and Hochberg, Y. (2000). On the adaptive control of the false discovery rate in multiple testing with independent statistics. J. Educ. Behav. Stat.

Beudel, M., and Brown, P. (2016). Adaptive deep brain stimulation in Parkinson's disease. Park. Relat. Disord.

Blankertz, B., Dornhege, G., Lemm, S., Krauledat, M., Curio, G., and Müller, K.R. (2006). The Berlin brain-computer interface: Machine learning based detection of user specific brain states. J. Univers. Comput. Sci.

Brainard, D.H. (1997). The Psychophysics Toolbox. Spat. Vis.

Breiman, L. (2001). Random forests. Mach. Learn.

Cagnan, H., Denison, T., Mclntyre, C., and Brown, P. (2019). Emerging technologies for improved deep brain stimulation. Nat Biotechnol 37, 1024-1033.

Caire, F., Ranoux, D., Guehl, D., Burbaud, P., and Cuny, E. (2013). A systematic review of studies on anatomical position of electrode contacts used for chronic subthalamic stimulation in Parkinson's disease. Acta Neurochir. (Wien).

Castaño-Candamil, S., Ferleger, B.I., Haddock, A., Cooper, S.S., Herron, J., Ko, A., Chizeck, H.J., and Tangermann, M. (2020). A Pilot Study on Data-Driven Adaptive Deep Brain Stimulation in Chronically Implanted Essential Tremor Patients. Front. Hum. Neurosci. 14, 541625.

Chen, T., and Guestrin, C. (2016). XGBoost: A scalable tree boosting system. In Proceedings of the ACM SIGKDD International Conference on Knowledge Discovery and Data Mining, $p$.

Cole, S.R., van der Meij, R., Peterson, E.J., de Hemptinne, C., Starr, P.A., and Voytek, B. (2017). Nonsinusoidal beta oscillations reflect cortical pathophysiology in parkinson's disease. J. Neurosci.

Dähne, S., Meinecke, F.C., Haufe, S., Höhne, J., Tangermann, M., Müller, K.R., and Nikulin, V. V. (2014). SPoC: A novel framework for relating the amplitude of neuronal oscillations to behaviorally relevant parameters. Neuroimage.

Dale, A.M., Fischl, B., and Sereno, M.I. (1999). Cortical surface-based analysis: I. Segmentation and surface reconstruction. Neuroimage.

Ewert, S., Plettig, P., Li, N., Chakravarty, M.M., Collins, D.L., Herrington, T.M., Kühn, A.A., and Horn, A. (2018). Toward defining deep brain stimulation targets in MNI space: A subcortical atlas based on multimodal MRI, histology and structural connectivity. Neuroimage.

Feldmann, L.K., Neumann, W., Krause, P., Lofredi, R., Schneider, G., and Kühn, A.A. (2021). Subthalamic beta band suppression reflects effective neuromodulation in chronic recordings. Eur. J. Neurol. ene.14801.

Ferleger, B.I., Houston, B., Thompson, M.C., Cooper, S.S., Sonnet, K.S., Ko, A.L., Herron, J.A., and Chizeck, H.J. (2020). Fully implanted adaptive deep brain stimulation in freely moving essential tremor patients. J. Neural Eng. 17, 056026. 
Fischer, P., Lipski, W.J., Neumann, W.J., Turner, R.S., Fries, P., Brown, P., and Mark Richardson, R. (2020). Movement-related coupling of human subthalamic nucleus spikes to cortical gamma. Elife.

Fisher, R.A. (1915). Frequency Distribution of the Values of the Correlation Coefficient in Samples from an Indefinitely Large Population. Biometrika.

Frazier, P.I. (2018). A tutorial on bayesian optimization. ArXiv.

Gall, J., and Lempitsky, V. (2013). Decision Forests for Computer Vision and Medical Image Analysis.

Geman, S., Bienenstock, E., and Doursat, R. (1992). Neural Networks and the Bias/Variance Dilemma. Neural Comput.

Gilron, R., Little, S., Perrone, R., Wilt, R., de Hemptinne, C., Yaroshinsky, M., Racine, C., Wang, S., Ostrem, J., Larson, P., et al. (2020). Chronic wireless streaming of invasive neural recordings at home for circuit discovery and adaptive stimulation. BioRxiv 2020.02.13.948349.

Gramfort, A., Luessi, M., Larson, E., Engemann, D.A., Strohmeier, D., Brodbeck, C., Goj, R., Jas, M., Brooks, T., Parkkonen, L., et al. (2013). MEG and EEG data analysis with MNE-Python. Front. Neurosci.

Gunduz, A., Brunner, P., Sharma, M., Leuthardt, E.C., Ritaccio, A.L., Pesaran, B., and Schalk, G. (2016). Differential roles of high gamma and local motor potentials for movement preparation and execution. Brain-Computer Interfaces.

Haller, M., Donoghue, T., Peterson, E., Varma, P., Sebastian, P., Gao, R., Noto, T., Knight, R., Shestyuk, A., and Voytek, B. (2018). Parameterizing neural power spectra. BioRxiv.

He, S., Debarros, J., Khawaldeh, S., Pogosyan, A., Mostofi, A., Baig, F., Pereira, E., Brown, P., and Tan, H. (2020). Closed-loop DBS triggered by real-time movement and tremor decoding based on thalamic LFPs for essential tremor. In Proceedings of the Annual International Conference of the IEEE Engineering in Medicine and Biology Society, EMBS, p.

He, S., Baig, F., Mostofi, A., Pogosyan, A., Debarros, J., Green, A.L., Aziz, T.Z., Pereira, E., Brown, P., and Tan, H. (2021). Closed-Loop Deep Brain Stimulation for Essential Tremor Based on Thalamic Local Field Potentials. Mov. Disord. mds.28513.

De Hemptinne, C., Swann, N.C., Ostrem, J.L., Ryapolova-Webb, E.S., San Luciano, M., Galifianakis, N.B., and Starr, P.A. (2015). Therapeutic deep brain stimulation reduces cortical phase-amplitude coupling in Parkinson's disease. Nat. Neurosci.

Holdgraf, C., Appelhoff, S., Bickel, S., Bouchard, K., D’Ambrosio, S., David, O., Devinsky, O., Dichter, B., Flinker, A., Foster, B.L., et al. (2019). iEEG-BIDS, extending the Brain Imaging Data Structure specification to human intracranial electrophysiology. Sci. Data.

Horn, A., and Fox, M.D. (2020). Opportunities of connectomic neuromodulation. Neuroimage.

Horn, A., Kühn, A.A., Merkl, A., Shih, L., Alterman, R., and Fox, M. (2017a). Probabilistic conversion of neurosurgical DBS electrode coordinates into MNI space. Neuroimage.

Horn, A., Reich, M., Vorwerk, J., Li, N., Wenzel, G., Fang, Q., Schmitz-Hübsch, T., Nickl, R., Kupsch, A., Volkmann, J., et al. (2017b). Connectivity Predicts deep brain stimulation outcome in Parkinson disease. Ann. Neurol.

Horn, A., Neumann, W.-J., Degen, K., Schneider, G.-H., and Kühn, A.A. (2017c). Toward an electrophysiological "sweet spot" for deep brain stimulation in the subthalamic nucleus. Hum. Brain Mapp. 
Horn, A., Li, N., Dembek, T.A., Kappel, A., Boulay, C., Ewert, S., Tietze, A., Husch, A., Perera, T., Neumann, W.J., et al. (2019). Lead-DBS v2: Towards a comprehensive pipeline for deep brain stimulation imaging. Neuroimage.

Houston, B., Thompson, M., Ko, A., and Chizeck, H. (2019). A machine-learning approach to volitional control of a closed-loop deep brain stimulation system. J. Neural Eng. 16.

Hwang, B.Y., Salimpour, Y., Tsehay, Y.K., Anderson, W.S., and Mills, K.A. (2020). Perspective: Phase Amplitude Coupling-Based Phase-Dependent Neuromodulation in Parkinson's Disease. Front. Neurosci.

Kehnemouyi, Y.M., Wilkins, K.B., Anidi, C.M., Anderson, R.W., Afzal, M.F., and Bronte-Stewart, H.M. (2021). Modulation of beta bursts in subthalamic sensorimotor circuits predicts improvement in bradykinesia. Brain 144, 473-486.

Khawaldeh, S., Tinkhauser, G., Shah, S.A., Peterman, K., Debove, I., Khoa Nguyen, T.A., Nowacki, A., Lenard Lachenmayer, M., Schuepbach, M., Pollo, C., et al. (2020). Subthalamic nucleus activity dynamics and limb movement prediction in Parkinson's disease. Brain.

Kingma, D.P., and Ba, J.L. (2015). Adam: A method for stochastic optimization. In 3rd International Conference on Learning Representations, ICLR 2015 - Conference Track Proceedings, p.

Kondylis, E.D., Randazzo, M.J., Alhourani, A., Lipski, W.J., Wozny, T.A., Pandya, Y., Ghuman, A.S., Turner, R.S., Crammond, D.J., and Richardson, R.M. (2016). Movement-related dynamics of cortical oscillations in Parkinson's disease and essential tremor. Brain.

Krauss, J.K., Lipsman, N., Aziz, T., Boutet, A., Brown, P., Chang, J.W., Davidson, B., Grill, W.M., Hariz, M.I., Horn, A., et al. (2021). Technology of deep brain stimulation: current status and future directions. Nat. Rev. Neurol.

Kühn, A.A., and Volkmann, J. (2017). Innovations in deep brain stimulation methodology. Mov. Disord.

Kühn, A.A., Williams, D., Kupsch, A., Limousin, P., Hariz, M., Schneider, G.H., Yarrow, K., and Brown, $P$. (2004). Event-related beta desynchronization in human subthalamic nucleus correlates with motor performance. Brain 127, 735-746.

Kühn, A.A., Kupsch, A., Schneider, G.H., and Brown, P. (2006). Reduction in subthalamic 8-35 Hz oscillatory activity correlates with clinical improvement in Parkinson's disease. Eur. J. Neurosci.

Leuthardt, E.C., Schalk, G., Wolpaw, J.R., Ojemann, J.G., and Moran, D.W. (2004). A brain-computer interface using electrocorticographic signals in humans. J. Neural Eng.

Li, N., Baldermann, J.C., Kibleur, A., Treu, S., Akram, H., Elias, G.J.B., Boutet, A., Lozano, A.M., AlFatly, B., Strange, B., et al. (2020). A unified connectomic target for deep brain stimulation in obsessivecompulsive disorder. Nat. Commun.

Little, S., Pogosyan, A., Neal, S., Zavala, B., Zrinzo, L., Hariz, M., Foltynie, T., Limousin, P., Ashkan, K., FitzGerald, J., et al. (2013). Adaptive deep brain stimulation in advanced Parkinson disease. Ann. Neurol. 74, 449-457.

Little, S., Bonaiuto, J., Barnes, G., and Bestmann, S. (2019). Human motor cortical beta bursts relate to movement planning and response errors. PLoS Biol.

Lofredi, R., Neumann, W.-J., Bock, A., Horn, A., Huebl, J., Siegert, S., Schneider, G.-H., Krauss, J.K., and Kühn, A.A. (2018). Dopamine-dependent scaling of subthalamic gamma bursts with movement velocity in patients with Parkinson's disease. Elife 7. 
Lofredi, R., Tan, H., Neumann, W.J., Yeh, C.H., Schneider, G.H., Kühn, A.A., and Brown, P. (2019). Beta bursts during continuous movements accompany the velocity decrement in Parkinson's disease patients. Neurobiol. Dis.

Lofredi, R., Auernig, G.C., Irmen, F., Nieweler, J., Neumann, W.-J., Horn, A., Schneider, G.-H., and Kühn, A.A. (2020). Subthalamic stimulation impairs stopping of ongoing movements. Brain.

Modarres, R., and Good, P. (1995). Permutation Tests: A Practical Guide to Resampling Methods for Testing Hypotheses. J. Am. Stat. Assoc.

Neumann, W., and Rodriguez-Oroz, M.C. (2021). Machine Learning Will Extend the Clinical Utility of Adaptive Deep Brain Stimulation. Mov. Disord. 36, 796-799.

Neumann, W.-J., Degen, K., Schneider, G.-H., Brücke, C., Huebl, J., Brown, P., and Kühn, A.A. (2016a). Subthalamic synchronized oscillatory activity correlates with motor impairment in patients with Parkinson's disease. Mov. Disord. 31, 1748-1751.

Neumann, W.-J.J., Schroll, H., de Almeida Marcelino, A.L., Horn, A., Ewert, S., Irmen, F., Krause, P., Schneider, G.-H.H., Hamker, F., and Kühn, A.A. (2018). Functional segregation of basal ganglia pathways in Parkinson's disease. Brain 141, 2655-2669.

Neumann, W.J., Degen, K., Schneider, G.H., Brücke, C., Huebl, J., Brown, P., and Kühn, A.A. (2016b). Subthalamic synchronized oscillatory activity correlates with motor impairment in patients with Parkinson's disease. Mov. Disord.

Neumann, W.J., Turner, R.S., Blankertz, B., Mitchell, T., Kühn, A.A., and Richardson, R.M. (2019a). Toward Electrophysiology-Based Intelligent Adaptive Deep Brain Stimulation for Movement Disorders. Neurotherapeutics.

Neumann, W.J., Turner, R.S., Blankertz, B., Mitchell, T., Kühn, A.A., and Richardson, R.M. (2019b). Toward Electrophysiology-Based Intelligent Adaptive Deep Brain Stimulation for Movement Disorders. Neurotherapeutics 16, 105-118.

Opri, E., Cernera, S., Molina, R., Eisinger, R.S., Cagle, J.N., Almeida, L., Denison, T., Okun, M.S., Foote, K.D., and Gunduz, A. (2020a). Chronic embedded cortico-thalamic closed-loop deep brain stimulation for the treatment of essential tremor. Sci. Transl. Med.

Opri, E., Cernera, S., Molina, R., Eisinger, R.S., Cagle, J.N., Almeida, L., Denison, T., Okun, M.S., Foote, K.D., and Gunduz, A. (2020b). Chronic embedded cortico-thalamic closed-loop deep brain stimulation for the treatment of essential tremor. Sci. Transl. Med. 12.

Pedregosa, F., Varoquaux, G., Gramfort, A., Michel, V., Thirion, B., Grisel, O., Blondel, M., Prettenhofer, P., Weiss, R., Dubourg, V., et al. (2011). Scikit-learn: Machine learning in Python. J. Mach. Learn. Res.

Petrucci, M.N., Neuville, R.S., Afzal, M.F., Velisar, A., Anidi, C.M., Anderson, R.W., Parker, J.E., O’Day, J.J., Wilkins, K.B., and Bronte-Stewart, H.M. (2020). Neural closed-loop deep brain stimulation for freezing of gait. Brain Stimul. 13, 1320-1322.

Piña-Fuentes, D., van Dijk, J.M.C., and M, B. (2019). Adaptive DBS in Parkinson's disease: Headlines, perspectives and challenges. Brain Stimul. 12, 1091-1092.

Proakis, J.G., and Monolakis, D.G. (1996). Digital signal processing: principles, algorithms, and applications.

Randazzo, M.J., Kondylis, E.D., Alhourani, A., Wozny, T.A., Lipski, W.J., Crammond, D.J., and Richardson, R.M. (2016). Three-dimensional localization of cortical electrodes in deep brain stimulation 
surgery from intraoperative fluoroscopy. Neuroimage.

Rosset, A., Spadola, L., and Ratib, O. (2004). OsiriX: An open-source software for navigating in multidimensional DICOM images. J. Digit. Imaging.

Schalk, G., Kubánek, J., Miller, K.J., Anderson, N.R., Leuthardt, E.C., Ojemann, J.G., Limbrick, D., Moran, D., Gerhardt, L.A., and Wolpaw, J.R. (2007). Decoding two-dimensional movement trajectories using electrocorticographic signals in humans. J. Neural Eng.

Schapire, R.E. (2009). A Short Introduction to Boosting. Society.

Schirrmeister, R.T., Springenberg, J.T., Dominique, L., Fiederer, J., Glasstetter, M., Eggensperger, K., Tangermann, M., Hutter, F., Burgard, W., and Ball, T. (2018). Deep learning with convolutional neural networks for brain mapping and decoding of movement-related information from the human EEG Short title: Convolutional neural networks in EEG analysis brain-machine interface $(\mathrm{BCl})$, brain-computer interface (BMI), model interpretability, brain mapping.

Shah, S.A., Tan, H., Tinkhauser, G., and Brown, P. (2018). Towards Real-Time, Continuous Decoding of Gripping Force from Deep Brain Local Field Potentials. IEEE Trans. Neural Syst. Rehabil. Eng. 26, 1460-1468.

Starr, P.A. (2018). Totally implantable bidirectional neural prostheses: A flexible platform for innovation in neuromodulation. Front. Neurosci.

Swann, N.C., De Hemptinne, C., Thompson, M.C., Miocinovic, S., Miller, A.M., Gilron, R., Ostrem, J.L., Chizeck, H.J., and Starr, P.A. (2018). Adaptive deep brain stimulation for Parkinson's disease using motor cortex sensing. J. Neural Eng.

Tan, H., Pogosyan, A., Ashkan, K., Green, A.L., Aziz, T., Foltynie, T., Limousin, P., Zrinzo, L., Hariz, M., and Brown, P. (2016). Decoding gripping force based on local field potentials recorded from subthalamic nucleus in humans.

Tan, H., Debarros, J., He, S., Pogosyan, A., Aziz, T.Z., Huang, Y., Wang, S., Timmermann, L., VisserVandewalle, V., Pedrosa, D.J., et al. (2019). Decoding voluntary movements and postural tremor based on thalamic LFPs as a basis for closed-loop stimulation for essential tremor. Brain Stimul. 12, 858-867.

Tinkhauser, G., Pogosyan, A., Little, S., Beudel, M., Herz, D.M., Tan, H., and Brown, P. (2017). The modulatory effect of adaptive deep brain stimulation on beta bursts in Parkinson's disease. Brain.

Torrecillos, F., Tinkhauser, G., Fischer, P., Green, A.L., Aziz, T.Z., Foltynie, T., Limousin, P., Zrinzo, L., Ashkan, K., Brown, P., et al. (2018). Modulation of beta bursts in the subthalamic nucleus predicts motor performance. J. Neurosci.

Turner, R.S., and Desmurget, M. (2010). Basal ganglia contributions to motor control: A vigorous tutor. Curr. Opin. Neurobiol.

Velisar, A., Syrkin-Nikolau, J., Blumenfeld, Z., Trager, M.H., Afzal, M.F., Prabhakar, V., and BronteStewart, H. (2019). Dual threshold neural closed loop deep brain stimulation in Parkinson disease patients. Brain Stimul. 12, 868-876.

Xie, Z., Schwartz, O., and Prasad, A. (2018). Decoding of finger trajectory from ECoG using deep learning. J. Neural Eng.

Yao, L., and Shoaran, M. (2019). Enhanced Classification of Individual Finger Movements with ECoG. In Conference Record - Asilomar Conference on Signals, Systems and Computers, p.

Zou, H., and Hastie, T. (2005). Regularization and variable selection via the elastic net. J. R. Stat. Soc. 
bioRxiv preprint doi: https://doi.org/10.1101/2021.04.24.441207; this version posted May 6, 2021. The copyright holder for this preprint (which was not certified by peer review) is the author/funder. All rights reserved. No reuse allowed without permission.

Ser. B Stat. Methodol. 
A

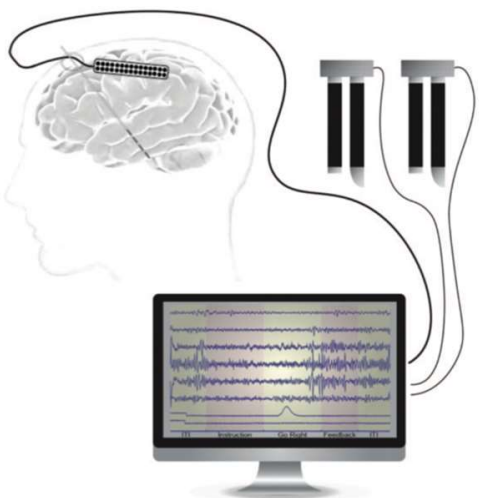

C
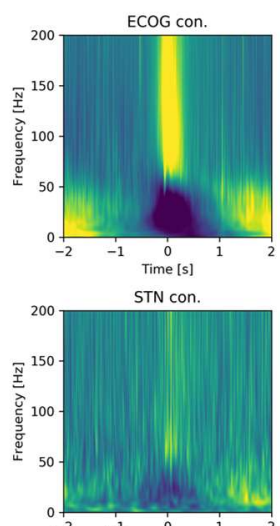

${ }^{-1} \stackrel{0}{\operatorname{Time}[s]}$
B

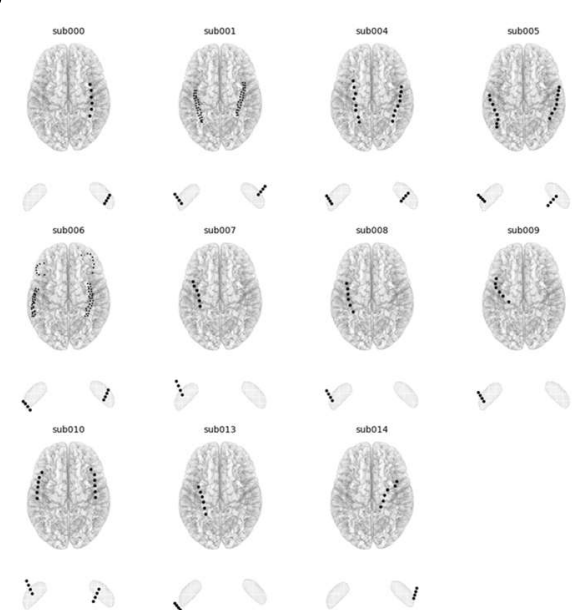

D

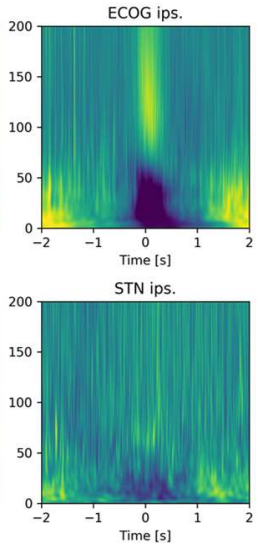

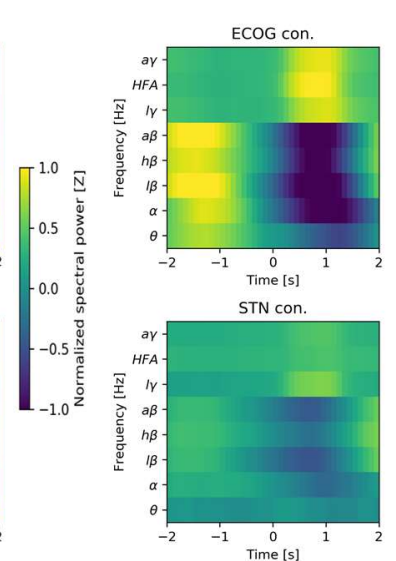

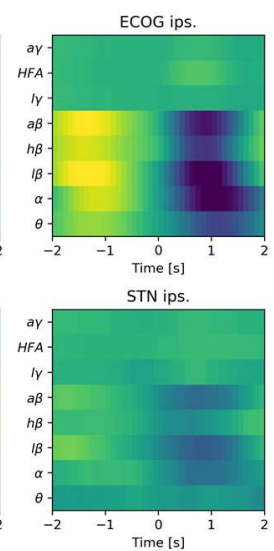

$E$
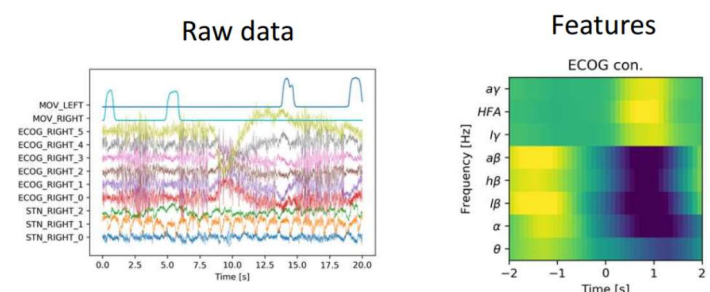

$R^{2}$ predictions
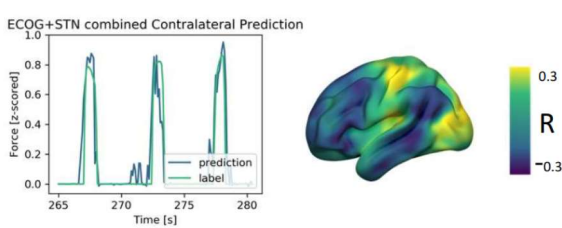

ᄂ

\begin{tabular}{|l|}
\hline \multicolumn{1}{|c|}{ Real Time processing pipeline [5] } \\
- ECOG Common Average \\
Referencing \\
- STN bipolar referencing \\
\hline \multicolumn{1}{|c|}{ Band Power Extraction } \\
\hline - Variance of bandpass \\
filtered windows \\
- Band power in different \\
frequency bands is \\
estimated via band specific \\
time windows \\
\multicolumn{1}{|c|}{ N } \\
\hline \multicolumn{1}{|c|}{ Normalization } \\
Median of previous $10 \mathrm{~s}$ \\
\hline
\end{tabular}

Robustly validated Machine Learning Pipeline for each channel [6]

Regression Problem

Prediction of Force Gripping

movement

Used Metric: $R^{2}$

Outer 3 fold Cross Validation

\begin{tabular}{|c|}
\hline Bayesian optimization \\
hyperparameter search \\
Inner 3 fold Cross Validation \\
\hline
\end{tabular}

\&

Functional and structural connectivity Analysis

- Leave one channel out prediction

- Leave one patient out

prediction

- SPM random effect analysis

\section{Localization}

- ECOG Localization [3]

- STN Localization [4]

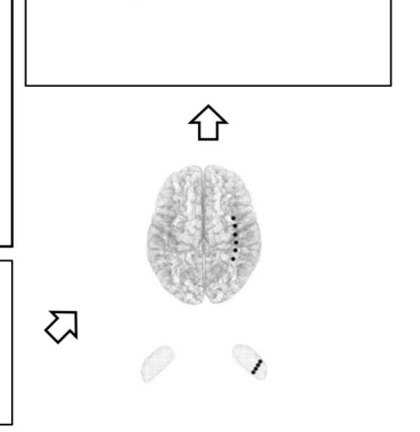


A
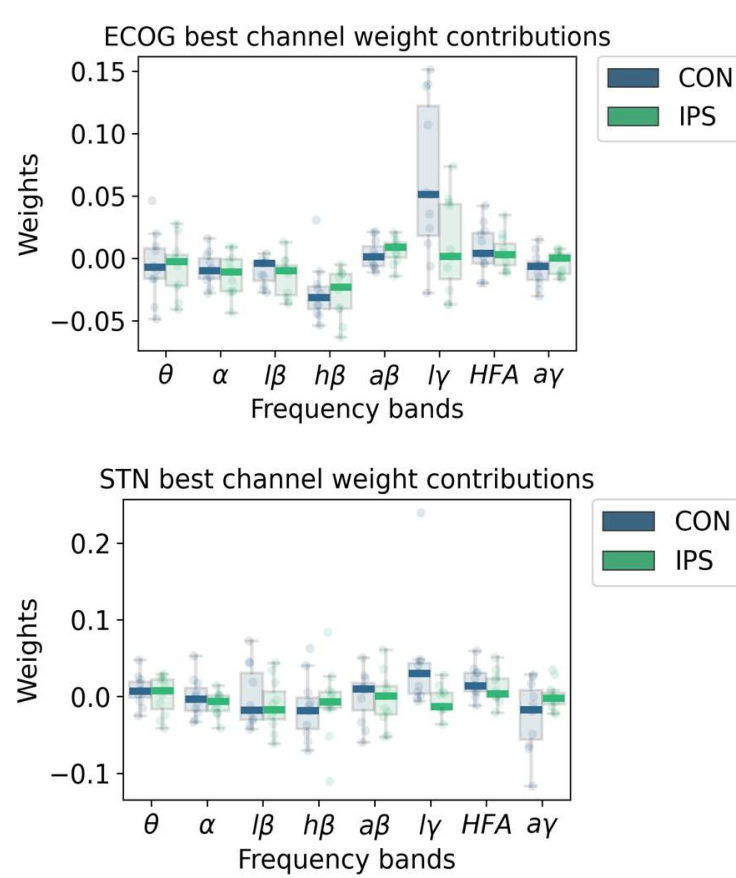

B
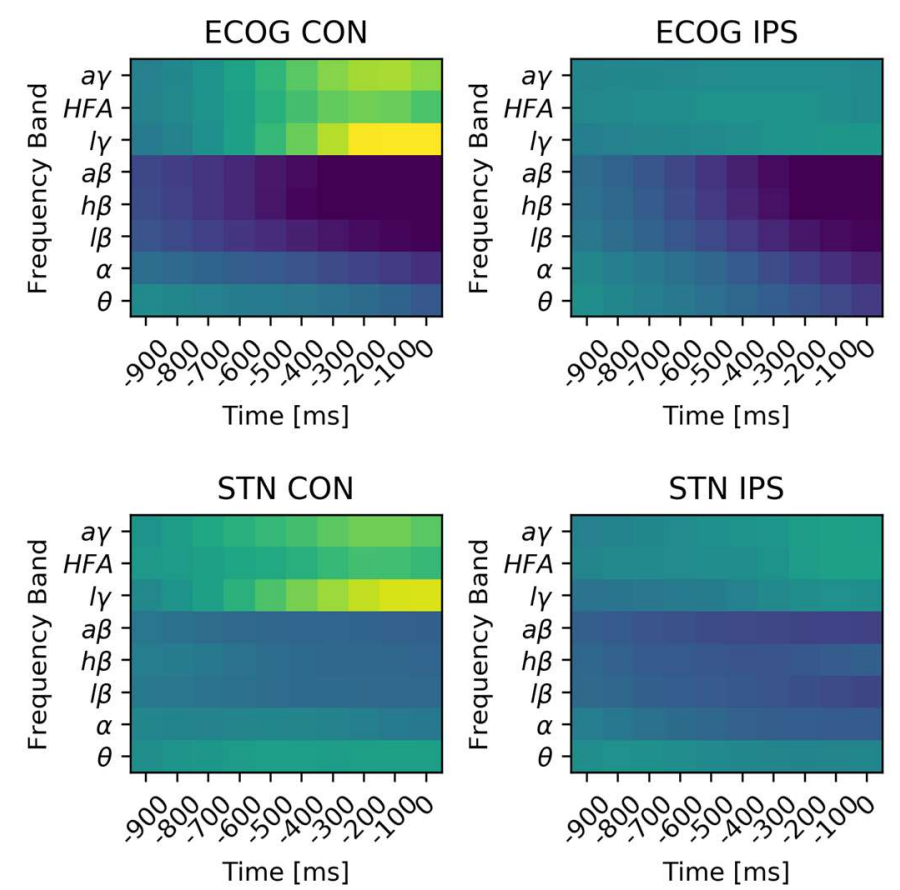

C

ECOG best channel Wiener Filter comparison

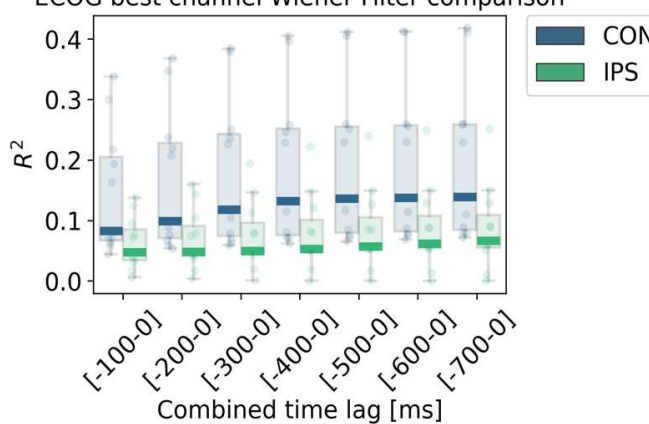

STN best channel Wiener Filter comparison

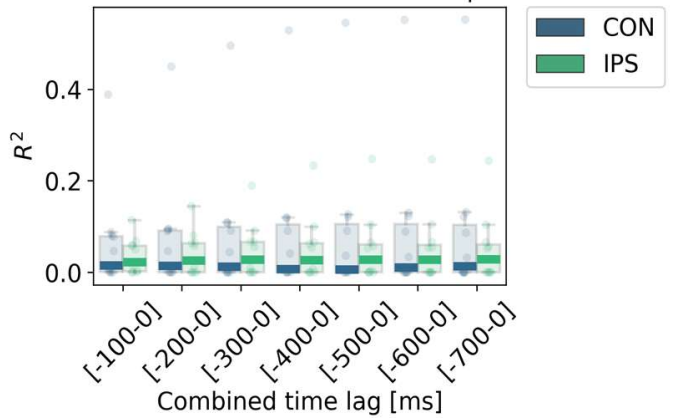



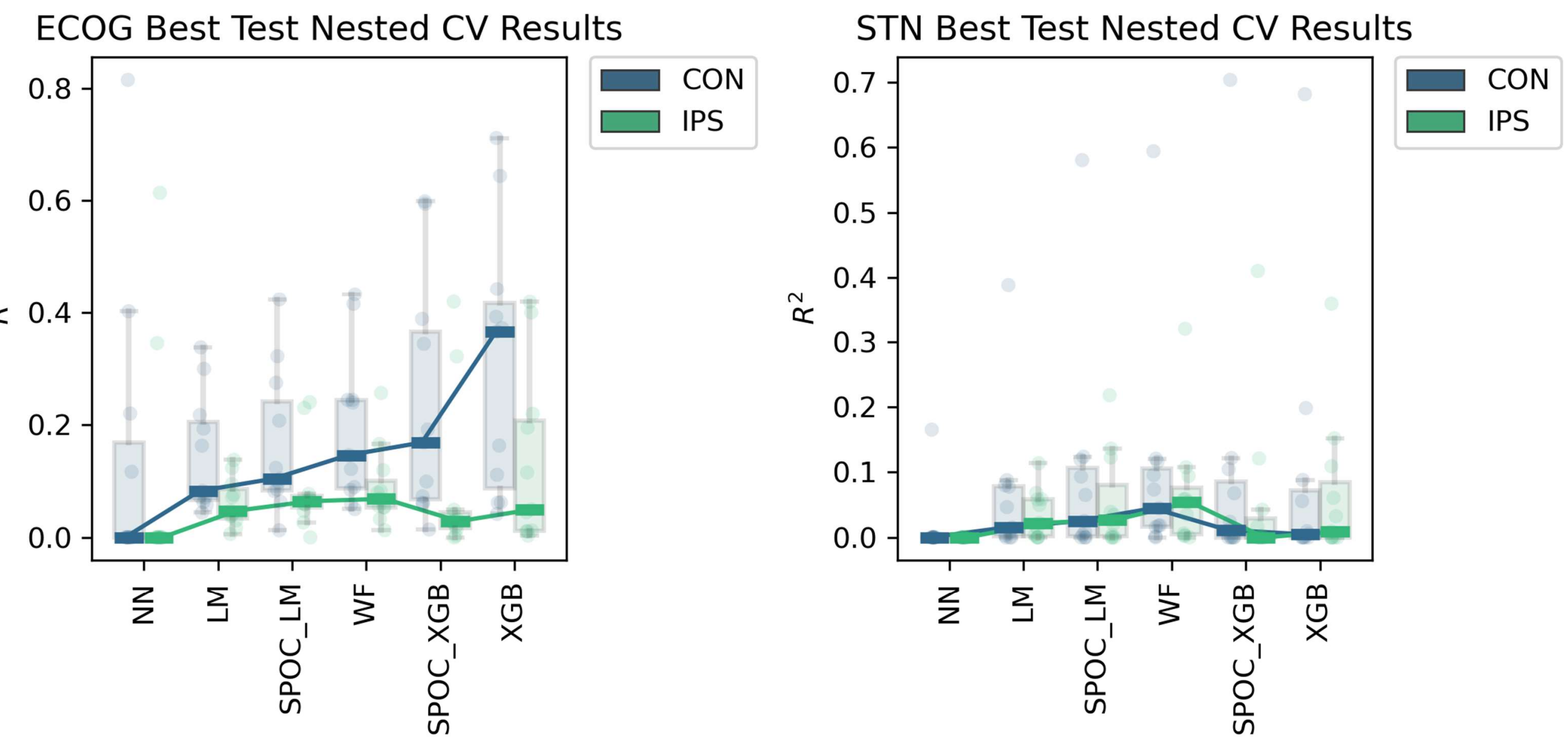
A

B

XGBOOST Cross Validation $R^{2}$ results

best ECOG + best STN con: $0.23 \pm 0.25$ ips: $0.16 \pm 0.21$

ECOG con: $0.31 \pm 0.24$ ips: $0.13 \pm 0.16$

STN con: $0.09 \pm 0.2$ ips: $0.07 \pm 0.11$

best ECOG vs best STN con. $p=0.0007$ ips. $p=0.0035$

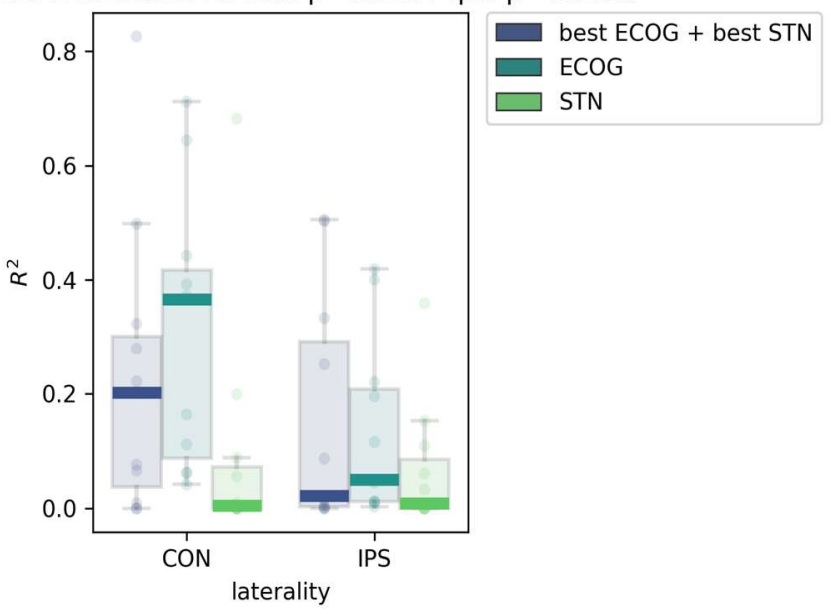

XGBOOST Cross Validation $R^{2}$ results

all ECOG + all STN con: $0.23 \pm 0.22$ ips: $0.14 \pm 0.18$

all ECOG con: $0.22 \pm 0.22$ ips: $0.12 \pm 0.16$

all STN con: $0.06 \pm 0.15$ ips: $0.04 \pm 0.07$

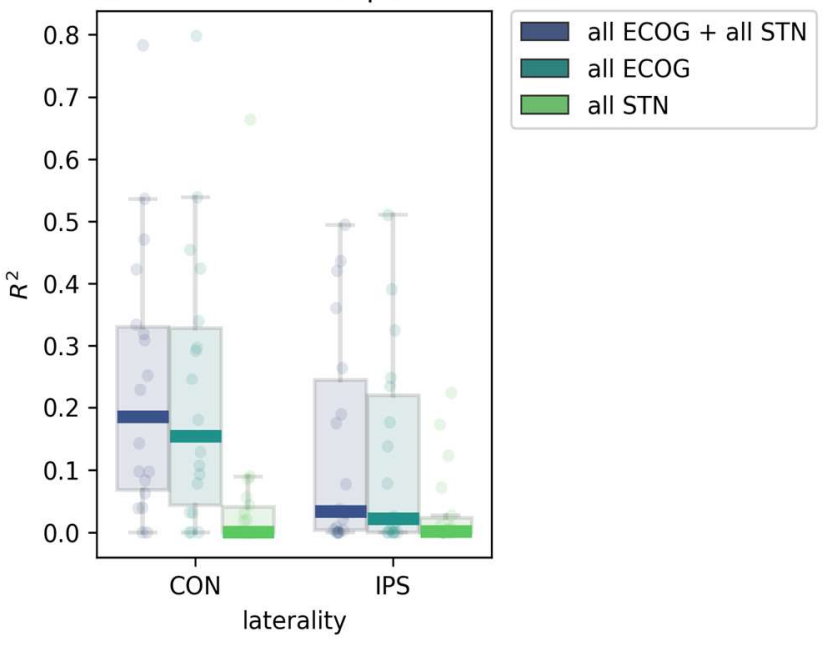

C

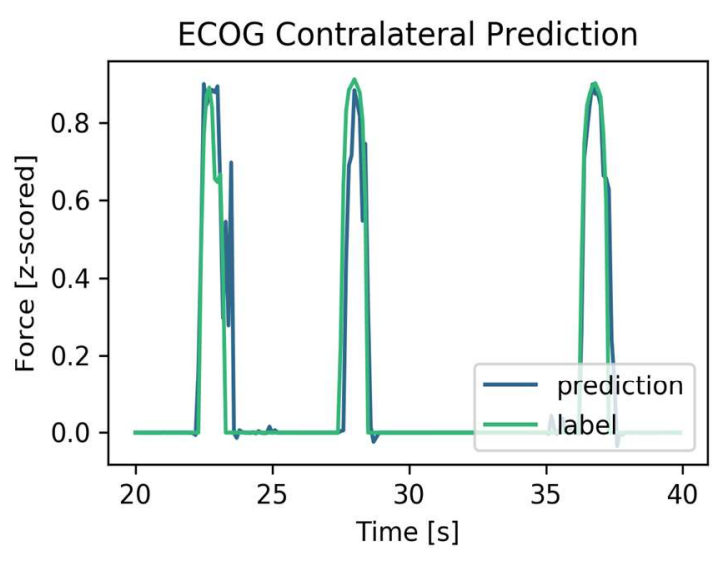


A

B

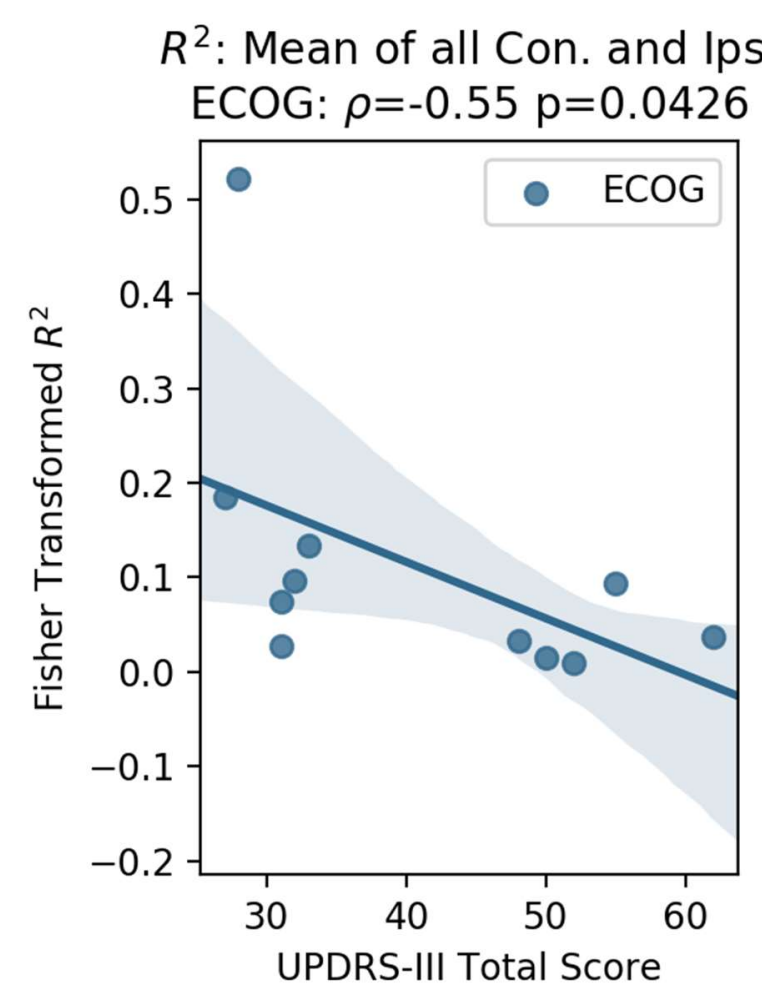

$R^{2}$ : Mean of Con. and Ips. STN: $\rho=-0.55 \mathrm{p}=0.0391$

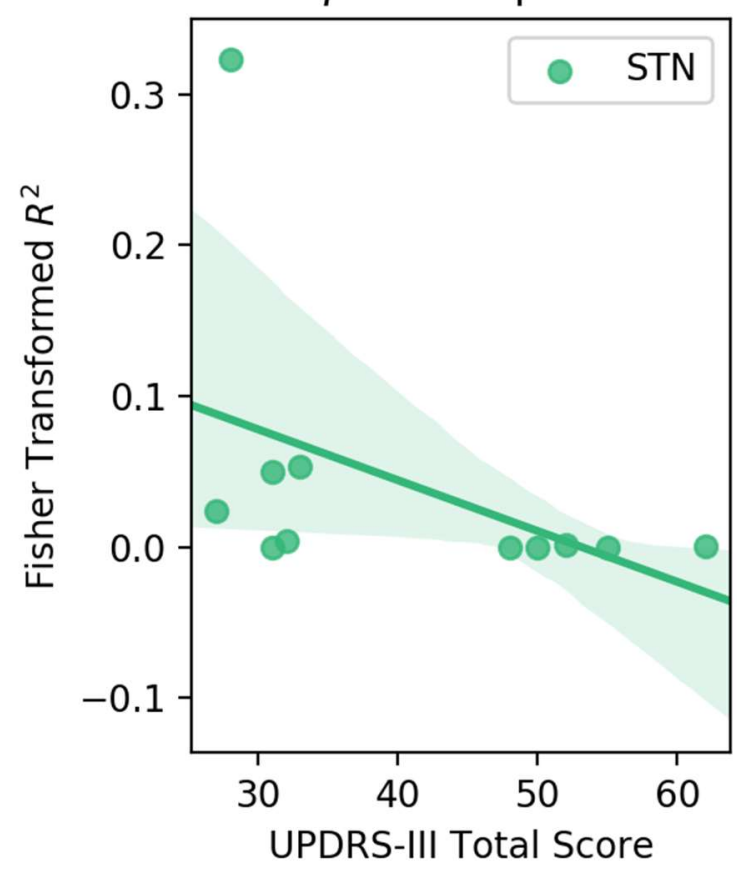

C

ECOG UPDRS-III total score correlation with mean prediction

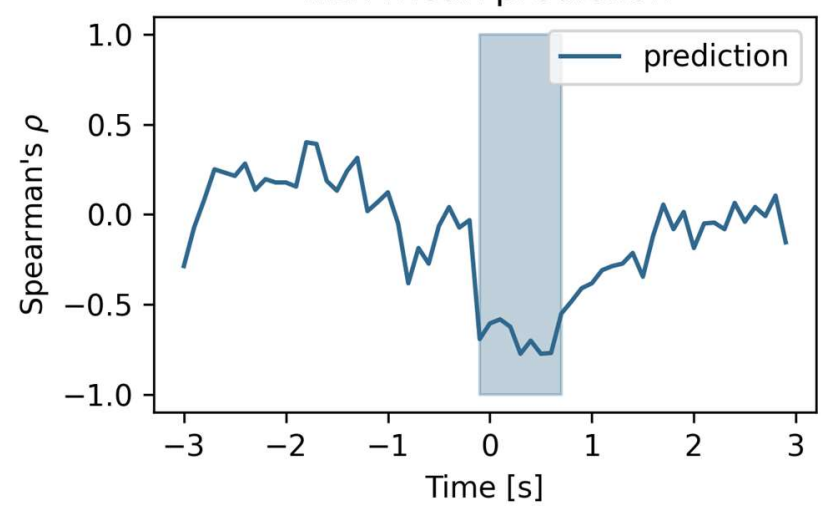

STN UPDRS-III total score correlation with mean prediction

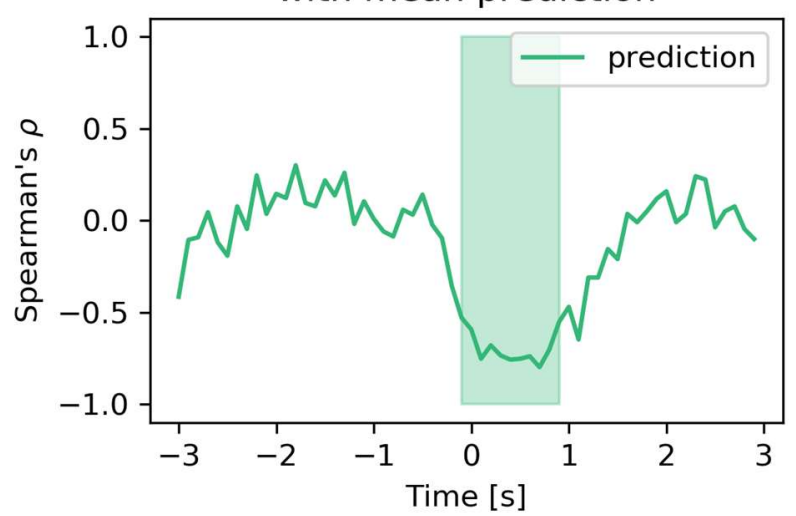


A

contralateral performance
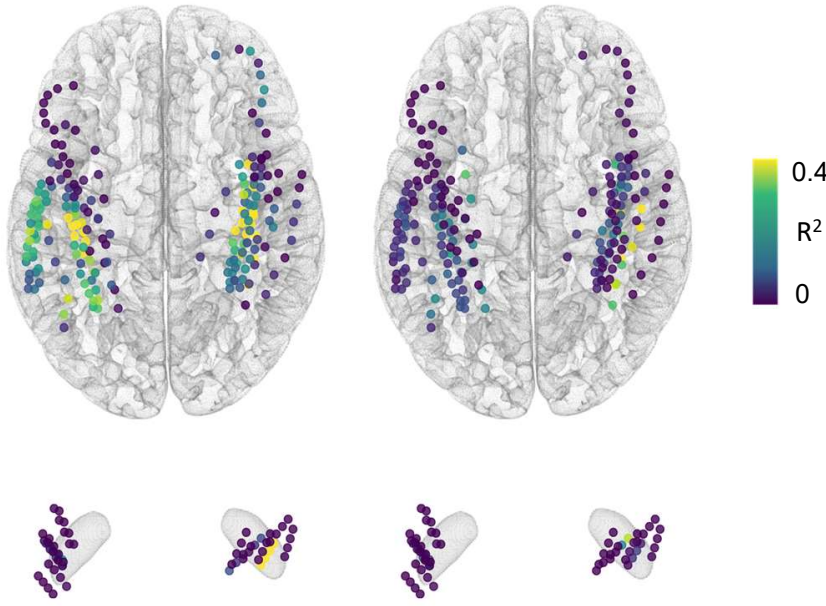

B

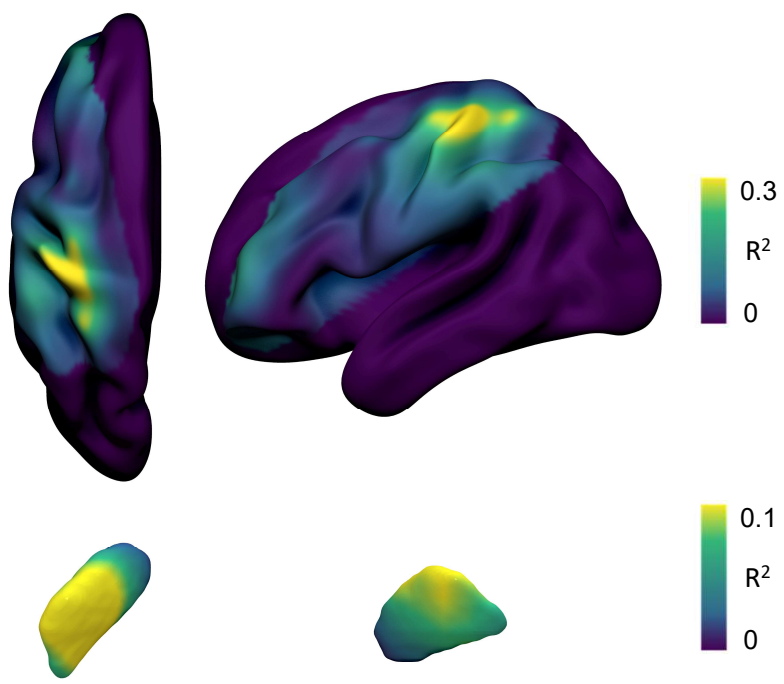




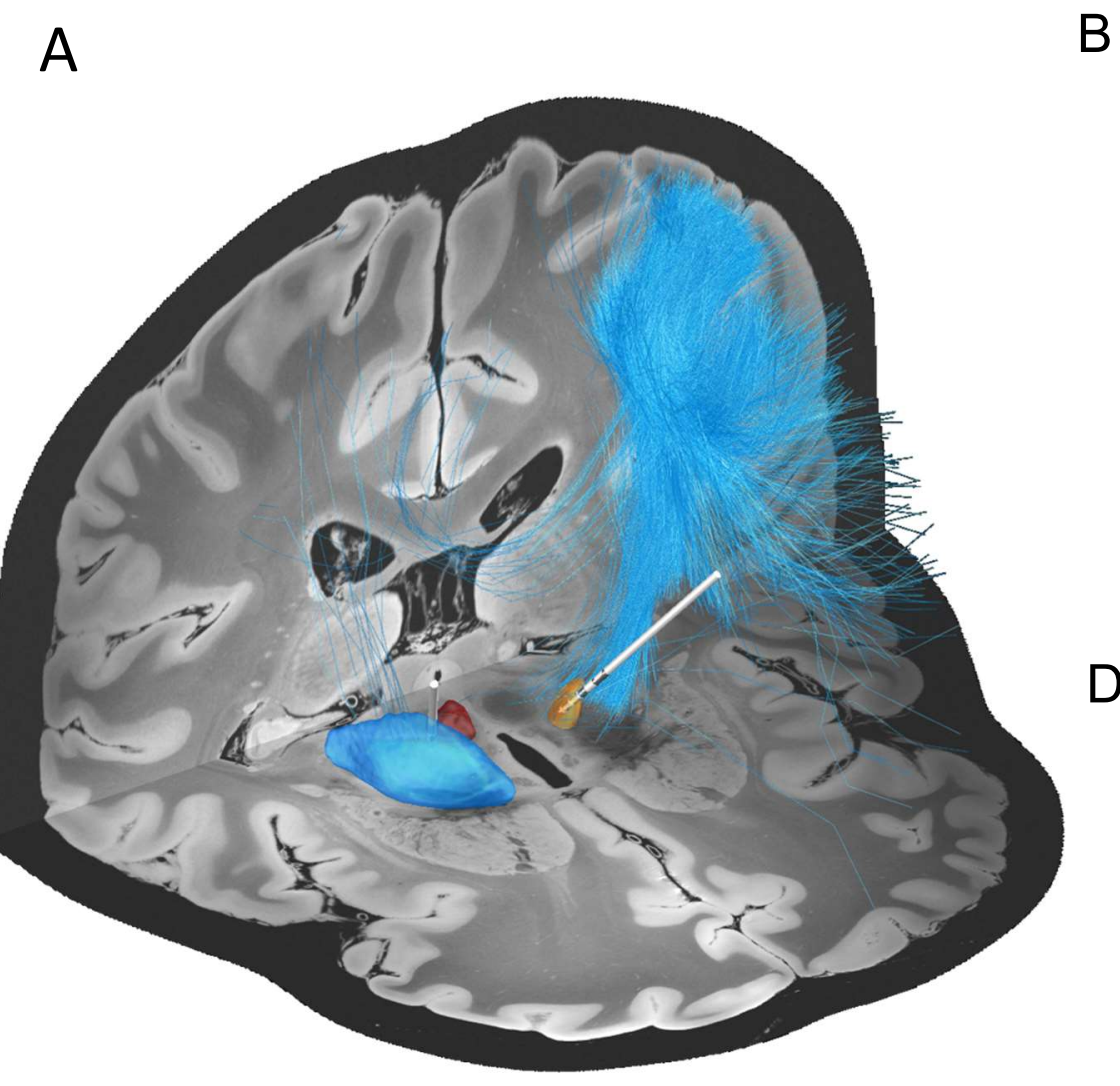

B

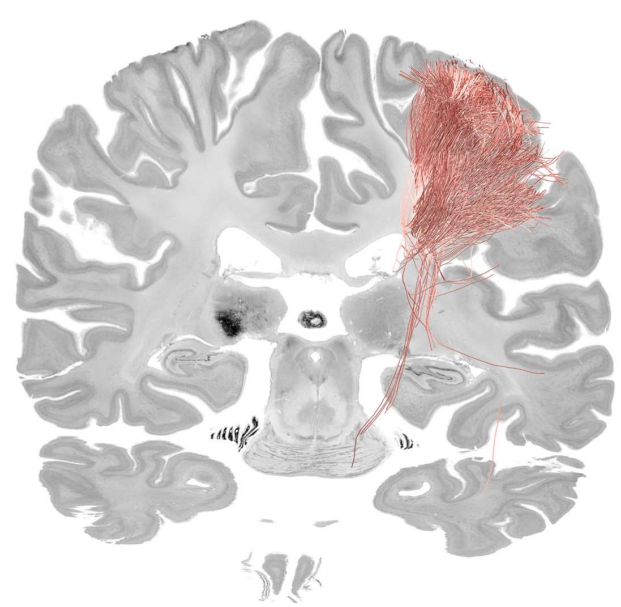

D

Fiber Tracking network decoding performance prediction Leave one channel out $\rho=0.38 p<0.0001$ Leave one patient out $\rho=0.24 p=0.0004$

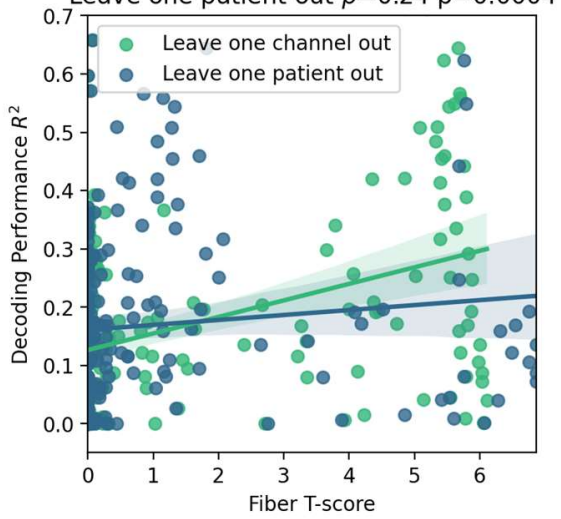

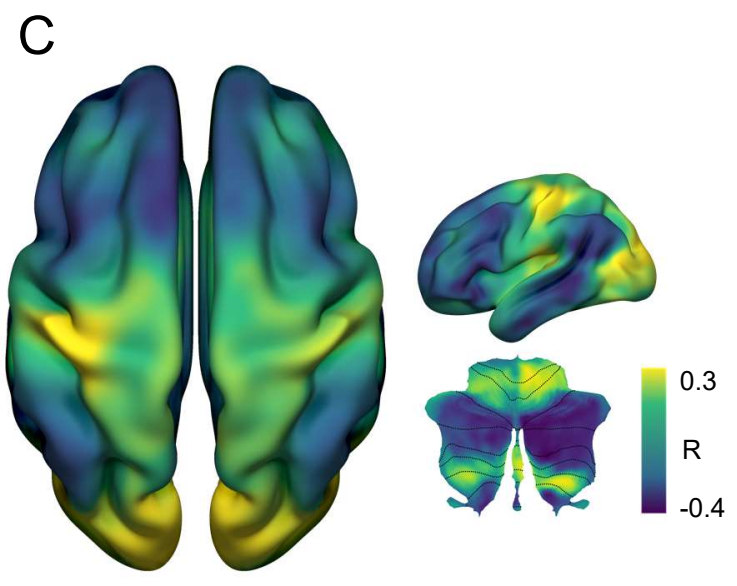

E

fMRI network decoding performance prediction Leave one channel out $\rho=0.37 p<0.0001$ Leave one patient out $\rho=0.25 \mathrm{p}=0.0004$

Leave one channel out $\because \infty$

0.6 Leave one patient out

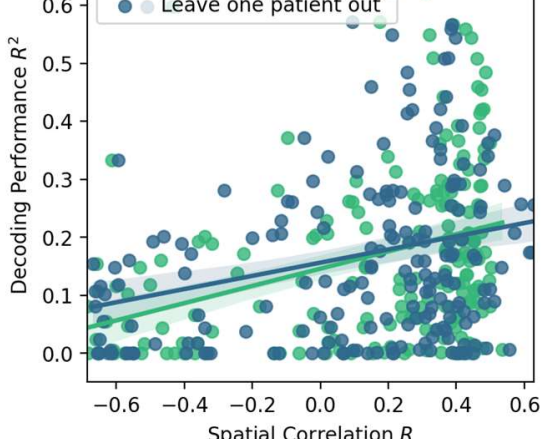

